\title{
Probing evolutionary population synthesis models in the near infrared with early-type galaxies
}

\author{
Luis Gabriel Dahmer-Hahn, ${ }^{1,2 \star}$ Rogério Riffel, ${ }^{1 \star}$ Alberto Rodríguez-Ardila, ${ }^{3}$ \\ Lucimara P. Martins, ${ }^{4}$ Carolina Kehrig, ${ }^{5}$ Timothy M. Heckman, ${ }^{6}$ Miriani G. Pastoriza ${ }^{1}$ \\ and Natacha Z. Dametto ${ }^{1}$ \\ ${ }^{1}$ Departamento de Astronomia, Universidade Federal do Rio Grande do Sul. AV. Bento Goncalves 9500, Porto Alegre, RS, Brazil \\ ${ }^{2}$ Instituto de Física e Química, Universidade Federal de Itajubá, Itajubá 37500-000, MG, Brazil \\ ${ }^{3}$ Laboratório Nacional de Astrofísica - Rua dos Estados Unidos 154, Bairro das Nações. CEP 37504-364, Itajubá, MG, Brazil \\ ${ }^{4}$ NAT - Universidade Cruzeiro do Sul, Rua Galvão Bueno, 868, São Paulo, SP, Brazil \\ ${ }^{5}$ Instituto de Astrofísica de Andalucía, CSIC, Apartado de correos 3004, E-18080 Granada, Spain \\ ${ }^{6}$ Center for Astrophysical Sciences, Department of Physics and Astronomy, The Johns Hopkins University, Baltimore, MD 21218, USA
}

Accepted 2018 February 22. Received 2018 January 27; in original form 2017 April 10

\begin{abstract}
We performed a near-infrared (NIR; $\sim 1.0-2.4 \mu \mathrm{m}$ ) stellar population study in a sample of early-type galaxies. The synthesis was performed using five different evolutionary population synthesis libraries of models. Our main results can be summarized as follows: low-spectral-resolution libraries are not able to produce reliable results when applied to the NIR alone, with each library finding a different dominant population. The two newest higher resolution models, on the other hand, perform considerably better, finding consistent results to each other and to literature values. We also found that optical results are consistent with each other even for lower resolution models. We also compared optical and NIR results and found out that lower resolution models tend to disagree in the optical and in the NIR, with higher fraction of young populations in the NIR and dust extinction $\sim 1$ mag higher than optical values. For higher resolution models, optical and NIR results tend to agree much better, suggesting that a higher spectral resolution is fundamental to improve the quality of the results.
\end{abstract}

Key words: stars: AGB and post-AGB - galaxies: stellar content - infrared: galaxies.

\section{INTRODUCTION}

Understanding the processes involved in galaxy evolution is one of the main topics of modern astrophysics. These processes are mainly driven by the star formation history (SFH) of the galaxies. One of the main methods to access the unresolved stellar content of galaxies is by comparing their observed spectra with combinations of simple stellar population (SSPs) libraries. These libraries can be empirical (in this case being limited by the properties of nearby stellar clusters) or can be constructed by using knowledge about stellar evolution, a technique called evolutionary population synthesis (EPS; e.g. Bruzual \& Charlot 2003; Maraston 2005; Conroy, Gunn \& White 2009; Meneses-Goytia et al. 2015; Röck et al. 2016, hereafter BC03, M05, C09, MG15, and MIUSCAT, respectively).

The SSPs are usually constructed using one of the two alternatives: isochrone synthesis or 'fuel consumption-based' algorithms. With the first approach, SSPs are calculated by integrating the stellar contributions to the flux in the various pass-bands of all mass-bins along one isochrone, after assuming an initial mass function (IMF; e.g. BC03). In the second approach, after leaving the main sequence, the duration of each subsequent phase in stellar evolution is calculated by using the fuel consumption theory (e.g. M05).

The big problem is that both approaches result in very different luminosities for short evolutionary stages, especially the crucial TP-AGB (thermally pulsing-asymptotic giant branch) phase, whose underlying physics is still poorly known (M05; Marigo et al. 2008; Conroy \& Gunn 2010; Kriek et al. 2010; Conroy 2013; Zibetti et al. 2013; Noël et al. 2013; Riffel et al. 2015). This happens because some processes of stellar evolution (mass-loss, changing opacities, dredge-up events, etc.) are not well understood, and receive a different treatment in each model flavour (e.g. BC03, M05, C09, MG15, and MIUSCAT). Models based on the fuel consumption theory tend to overpredict TP-AGB features while those based on isochrone synthesis generally underestimate them (Zibetti et al. 2013, and references therein), although a few models based on isochrone synthesis also contain large amounts of these stars (e.g. Marigo et al. 2008). Currently, there is no consensus 
Table 1. Basic properties for the sample.

\begin{tabular}{|c|c|c|c|c|c|c|c|c|}
\hline Object & Morphology ${ }^{a}$ & $K$ magnitude $^{b}$ & $\begin{array}{c}\text { Absolute } \\
K \text { magnitude }^{c}\end{array}$ & $z^{d}$ & $\begin{array}{l}\text { Aperture radius } \\
\quad(\operatorname{arcsec})\end{array}$ & $\begin{array}{l}\text { Exposure time } \\
\quad(\min )\end{array}$ & $\mathrm{S} / \mathrm{N}^{e}$ & $\begin{array}{l}\text { Optical }^{f} \\
\text { spectrum }\end{array}$ \\
\hline NGC 4636 & E0-1 & 9.0 & -21.6 & 0.003129 & 10.0 & 27.0 & 54 & SDSS \\
\hline NGC 5905 & $\mathrm{SB}(\mathrm{r}) \mathrm{b}$ & 11.0 & -22.4 & 0.011308 & 7.0 & 24.0 & 30 & - \\
\hline NGC 5966 & $\mathrm{E}$ & 10.7 & -23.3 & 0.014924 & 6.0 & 24.0 & 24 & CALIFA \\
\hline NGC 6081 & S0 & 10.5 & -23.8 & 0.017265 & 6.0 & 36.0 & 45 & CALIFA \\
\hline NGC 6146 & $\mathrm{E}$ ? & 10.3 & -25.2 & 0.029420 & 3.0 & 36.0 & 56 & CALIFA \\
\hline NGC 6338 & So & 10.6 & -24.7 & 0.027427 & 4.0 & 36.0 & 30 & CALIFA \\
\hline UGC 08234 & $\mathrm{~S} 0 / \mathrm{a}$ & 10.7 & -24.6 & 0.027025 & 6.0 & 36.0 & 32 & CALIFA \\
\hline
\end{tabular}

Notes. ${ }^{a}$ de Vaucouleurs et al. (1991); ${ }^{b}$ Two Micron All Sky Survey team, 2003, 2MASS extended objects, final release; ${ }^{c}$ Calculated based in the apparent magnitude and the redshift and assuming $H=70 \mathrm{~km} \mathrm{~s}^{-1} \mathrm{Mpc}^{-1} ;{ }^{d} \mathrm{NED} ;{ }^{e} K$-band signal-to-noise ratio before smoothing the spectra; ${ }^{f}$ Presence or absence of SDSS spectra.
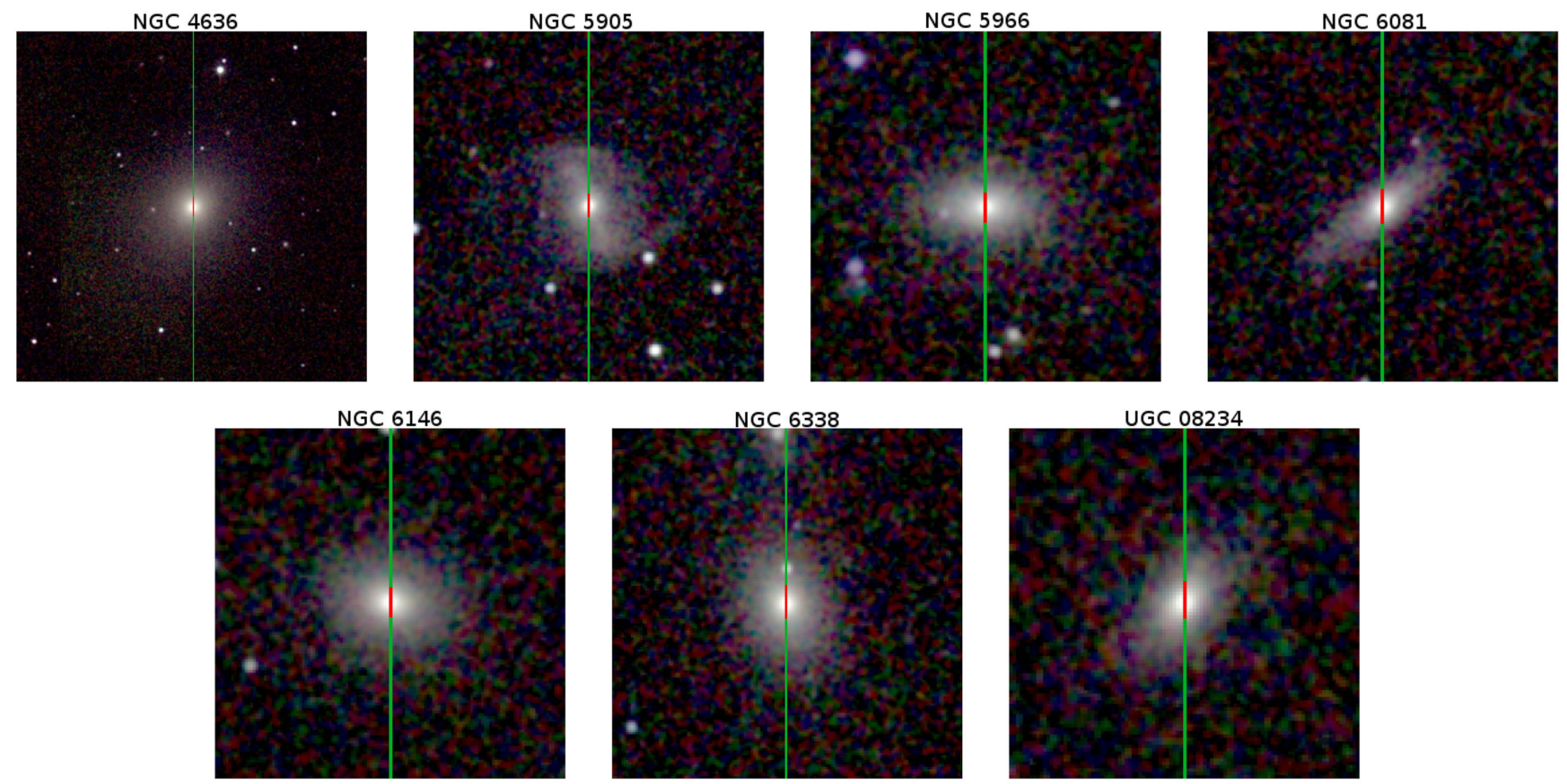

Figure 1. Combined 2MASS JHKs imaging for the galaxy sample. The vertical green line shows the slit orientation while the red segment indicates the aperture used to extract the spectra.

regarding what TP-AGB contribution best reproduces the observed spectra of galaxies. Many stellar absorption features predicted by TP-AGB heavy models have been found, like the $1.1 \mu \mathrm{m} \mathrm{CN}$ band (Riffel et al. 2007), the $1.4 \mu \mathrm{m} \mathrm{CN}$ band (Martins et al. 2013), and the $\mathrm{ZrO}$ features at 0.8-1.0 $\mu \mathrm{m}$ (Martins et al. 2013). On the other hand, Zibetti et al. (2013) did not detect the TP-AGB spectral features predicted by M05 in their spectra of post-starburst galaxies at $z \sim 0.2$. Riffel et al. (2015) found that models based on empirical libraries that predict relatively strong near-infrared (NIR) features provide a more accurate description of the data. However, none of the models tested by them successfully reproduces all of the features observed in the spectra. Also, Riffel et al. (2015) claimed that stars in other evolutionary phases like red giant branch (RGB) may be crucial to describe the absorption features detected in galaxies and that empirical spectra of these kind of stars should be included in the EPS models.

The libraries of SSPs are then used by a computing code (e.g. STARLIGHT; Cid Fernandes et al. 2005) to determine parameters such as ages, element abundances, stellar masses, and stellar mass functions by searching for the combination of SSPs that best reproduce the observed spectrum.

A major issue when characterizing the stellar population of a galaxy is attributed to the dependence of results in the model set used when fitting the underlying stellar features (Chen et al. 2010). This happens because of all the uncertainties related to the construction of the SSPs. Summed to these effects, there is the well-known agemetallicity degeneracy, which is difficult to distinguish between an old stellar population and a reddened or more metallic younger one (Worthey 1994).

Here we aim to compare the stellar population predictions derived using NIR spectra of different sets of EPS models for the central region of six local early-type galaxies (ETGs) and one spiral galaxy. A similar study has been published by Baldwin et al. (2018), who used 12 NIR spectra of early-type galaxies and tested four stellar population synthesis models. They found that the derived star formation histories vary dramatically from model to model 


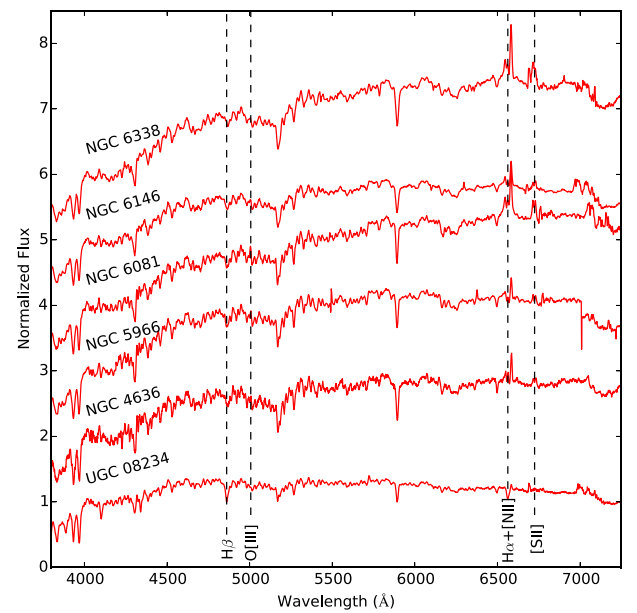

Figure 2. Red curves represent SDSS spectra, which are available for four galaxies of the sample. Rest-frame wavelengths of the main optical emissionlines are indicated.

in the near-infrared, and that this variation is largely dependent on the stellar library used to build the models and on the shape of the continuum. The former morphological type was chosen because it contains a relatively homogeneous old stellar population. We are aware, though, that current day interpretation is that these objects experienced moderately different star formation histories, with the present-day stellar populations slightly differing in metallicity and/or age (Rickes, Pastoriza \& Bonatto 2009, and references therein).

This paper is structured as follows: The data and reduction process are presented in Section 2. The stellar population synthesis method used in the analysis is presented in Section 3. In Section 4, we present the main results from the synthesis using the different sets of SSPs. A discussion of these results is in Section 5. The final remarks are given in Section 6.

\section{DATA AND REDUCTION}

From the six ETGs, five are from the Calar Alto Legacy Integral Field Area Survey (CALIFA; Sánchez et al. 2012; Walcher et al. 2014; Sánchez et al. 2016). These objects were selected for being the ETGs in the CALIFA data release 1 and accessible to the night sky during the observing run. Also, the CALIFA papers confirmed the presence of warm gas for these five galaxies. The final seven targets were selected on the basis of surface brightness to offer a good compromise between signal-to-noise ratio (S/N) and exposure time. We also observed NGC 4636, a typical LINER (lowionization nuclear emission-line region), and NGC 5905, classified as $\mathrm{SB}(\mathrm{r}) \mathrm{b}$, for comparison. The infrared data were obtained at the Astrophysical Research Consortium (ARC) telescope located at the Apache Point Observatory (APO). The TripleSpec (Wilson et al. 2004) instrument was used to obtain cross-dispersed spectra in the range $0.95-2.46 \mu \mathrm{m}$. We used the 1.1 arcsec slit, resulting in a spectral resolution of $R \sim 2000$. After each target, we observed an A0V, A1V, or A2V star at a similar airmass for flux calibration and telluric correction. Both the science objects and the telluric stars were observed following the dithering pattern object-sky-object. Internal flat-field and arc lamp exposures were also acquired for pixel response and wavelength calibration, respectively. The reduction of the data was done using TRIPLESPECTOOL, a modified version of Spextool (Cushing, Vacca \& Rayner 2004; Vacca, Cushing \&
Rayner 2004) using standard settings. These spectra are available for download at the MNRAS website.

Table 1 shows the basic properties for the sample. Also, in order to compare NIR results with optical ones, for the five CALIFA objects, we extracted optical spectra using apertures of size similar to that of the NIR slit. For NGC 4636, which is not within the CALIFA targets, we used optical spectra from Sloan Digital Sky Survey (SDSS). Fig. 1 shows Two Micron All Sky Survey (2MASS) JHK imaging of the sample with the slit orientation. The individual description of the objects of the sample is presented in Appendix A. Optical and NIR spectra are shown in Figs 2 and 3, respectively.

\section{STELLAR POPULATION SYNTHESIS}

The stellar population synthesis technique consists basically of comparing the observed spectrum of a galaxy with a combination of SSPs with different ages and metallicities (this set of SSPs is known as a base of elements; Cid Fernandes et al. 2005), searching for a combination of SSPs that suitably fits the observed spectrum.

For a proper fitting of a galaxy's stellar population, the library of models must cover the range of possible observed spectral properties (e.g. ages and metallicities). It is also fundamental to choose an adequate number of elements for such library in order to have nondegenerate solutions (Schmidt et al. 1991; Cid Fernandes et al. 2005; Dametto et al. 2014). Chen et al. (2010) presented a study in the optical region showing that different models may result in quite different SFHs for the same observed spectrum. Studying six different types of galaxies (star-forming galaxies, composite galaxies, Seyfert 2s, LINERs, E+A, and ETGs) using six different EPS models, they found that the differences found are significant, but the dominant populations are unaltered. Also, they found that using the same models, the results depend on the selected ages. In the NIR, this scenario seems to be more dramatic, since the inclusion of the TP-AGB phase in the models is still very uncertain and is a matter of debate (M05, Marigo et al. 2008; Conroy \& Gunn 2010; Kriek et al. 2010; Conroy 2013; Zibetti et al. 2013; Noël et al. 2013; Riffel et al. 2015). To properly address this issue we decided to build eight different libraries of models using five different EPS models flavours, as follows:

(i) Bruzual \& Charlot (2003, BC03)

(ii) Maraston (2005, M05)

(iii) Conroy et al. (2009, C09)

(iv) Meneses-Goytia et al. (2015, MG15)

(v) Röck et al. (2016, MIUSCAT)

Details of the EPS models, as well as the chosen ages, metallicities, and evolutionary tracks of the SSPs used in the fitting are listed in Table 2. Since MG15 and MIUSCAT have only SSPs with ages $t \geq 1 \mathrm{Gyr}$, in order to allow for a suitable comparison of the results from the different EPS models, we created three additional libraries of models by removing the SSPs with ages $t<1 \mathrm{Gyr}$ from BC03, M05, and C09 models. These libraries do not include SSPs younger than $1 \mathrm{Gyr}$ because of the lack of hot stars in the Infrared Telescope Facility (IRTF) library (Cushing, Rayner \& Vacca 2005; Rayner, Cushing \& Vacca 2009), which was used to build the models.

It is worth mentioning that in the NIR, the spectral resolution of $\mathrm{BC} 03, \mathrm{M} 05$, and $\mathrm{C} 09$ is lower than that of the observed spectra. We then rebinned the data in order to match the spectral resolution of the models. Considering that MG15 and MIUSCAT have a spectral resolution similar to that of the observed spectra, this procedure was not necessary when these later libraries were employed. 


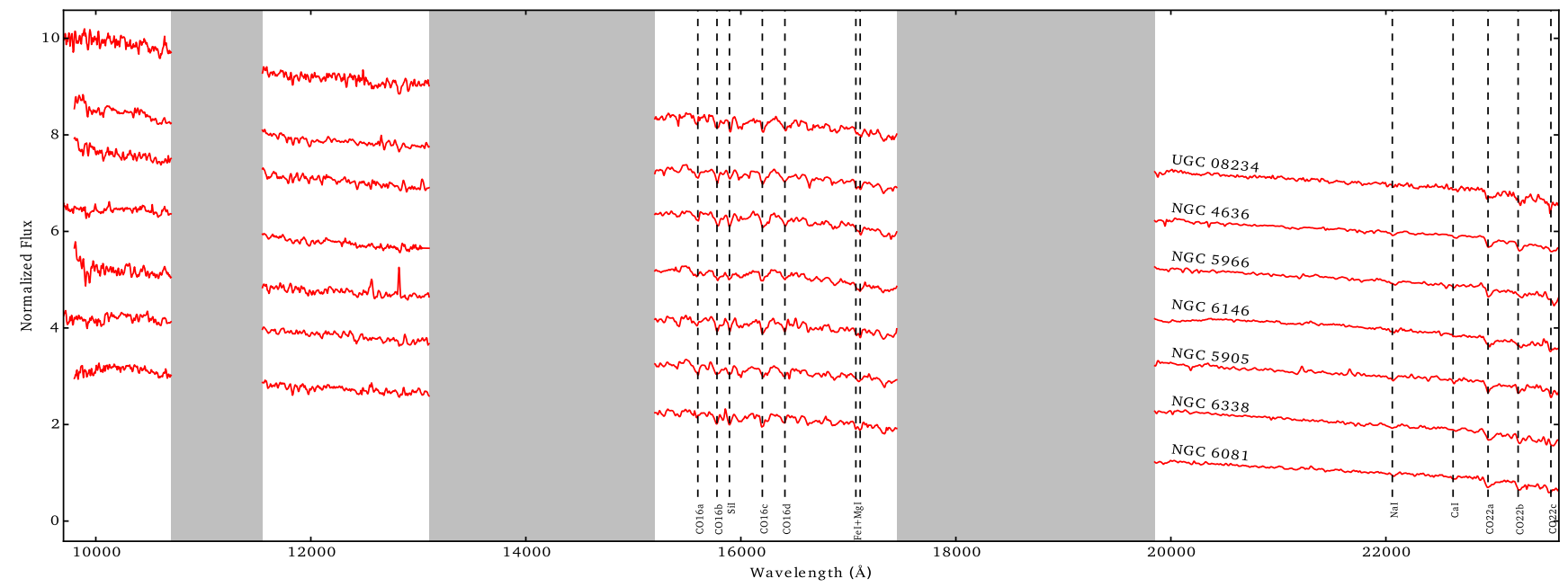

Figure 3. NIR spectra for the sample. The main absorption bands are indicated by dashed lines. High telluric absorption regions are shaded.

Table 2. Basic properties for the EPS models and information on the used SSPs.

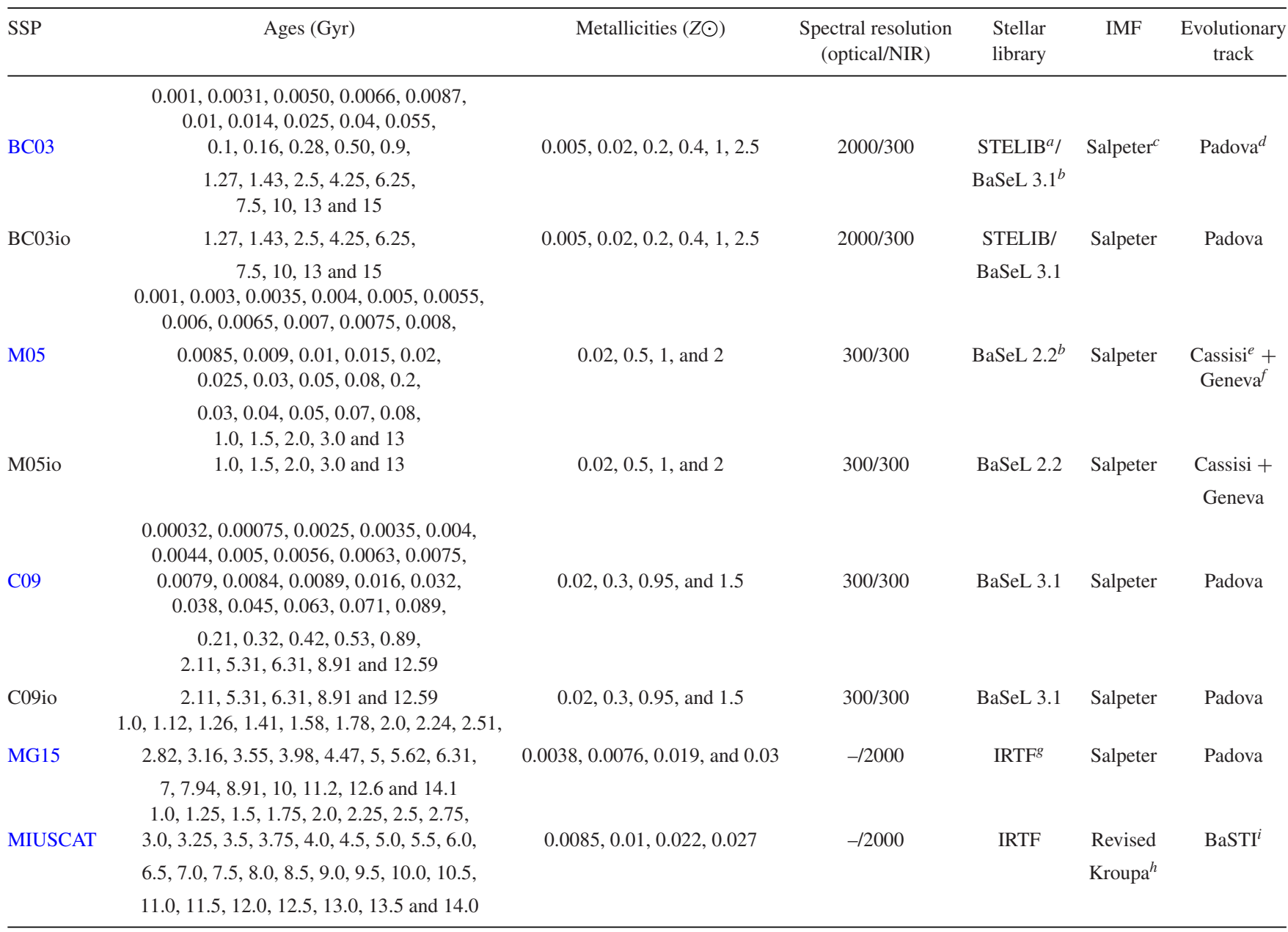

Notes. ${ }^{a}$ Le Borgne et al. (2003); ${ }^{b}$ Lejeune, Cuisinier \& Buser (1997, 1998), Westera et al. (2002); ${ }^{c}$ Salpeter (1955); ${ }^{d}$ Marigo et al. (2008) and references therein; ${ }^{e}$ Cassisi, Castellani \& Castellani (1997b), Cassisi, degl'Innocenti \& Salaris (1997a); ${ }^{f}$ Schaller et al. (1992); ${ }^{g}$ Cushing et al. (2005); Rayner et al. (2009); ${ }^{h}$ Kroupa (2001); ${ }^{i}$ Pietrinferni et al. (2004). 

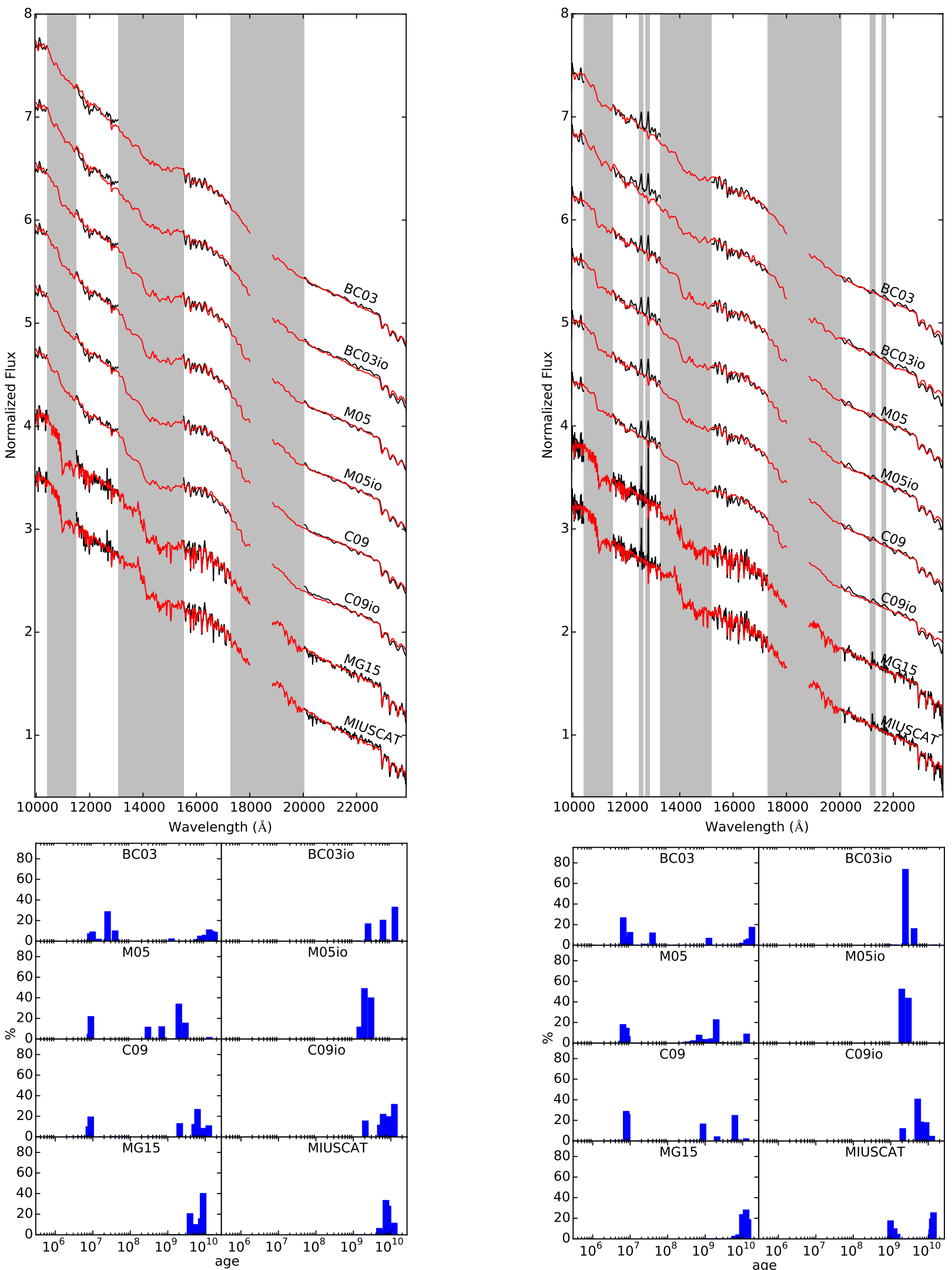

Figure 4. NIR results for NGC 4636 using eight libraries of models. On the upper panel, observed spectrum is shown in black and modelled spectrum is shown in red. Masked areas are shaded. On the bottom panel, we show in blue the percentage luminosity contribution for every age.

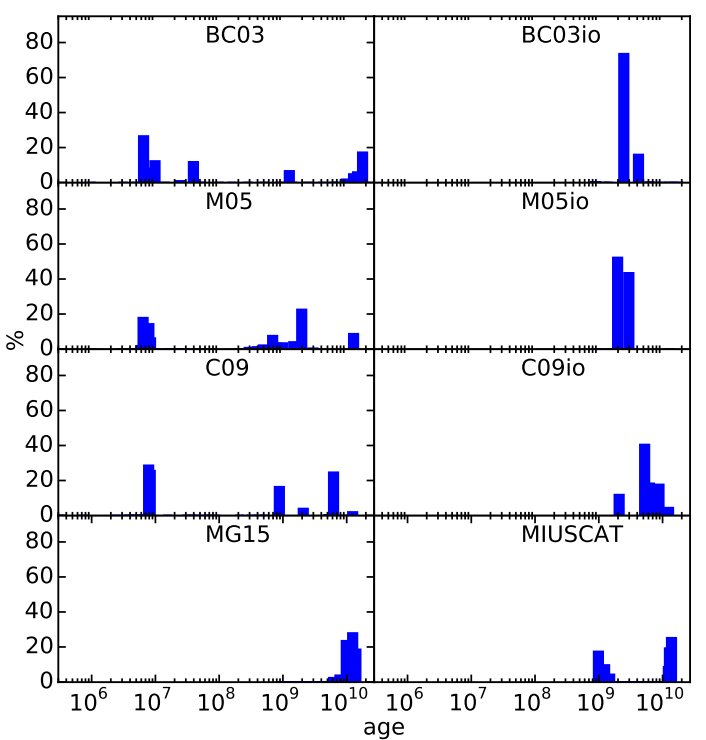

Figure 5. Same as Fig. 4 for NGC 5905. Emission lines are also shaded on the upper panel. 

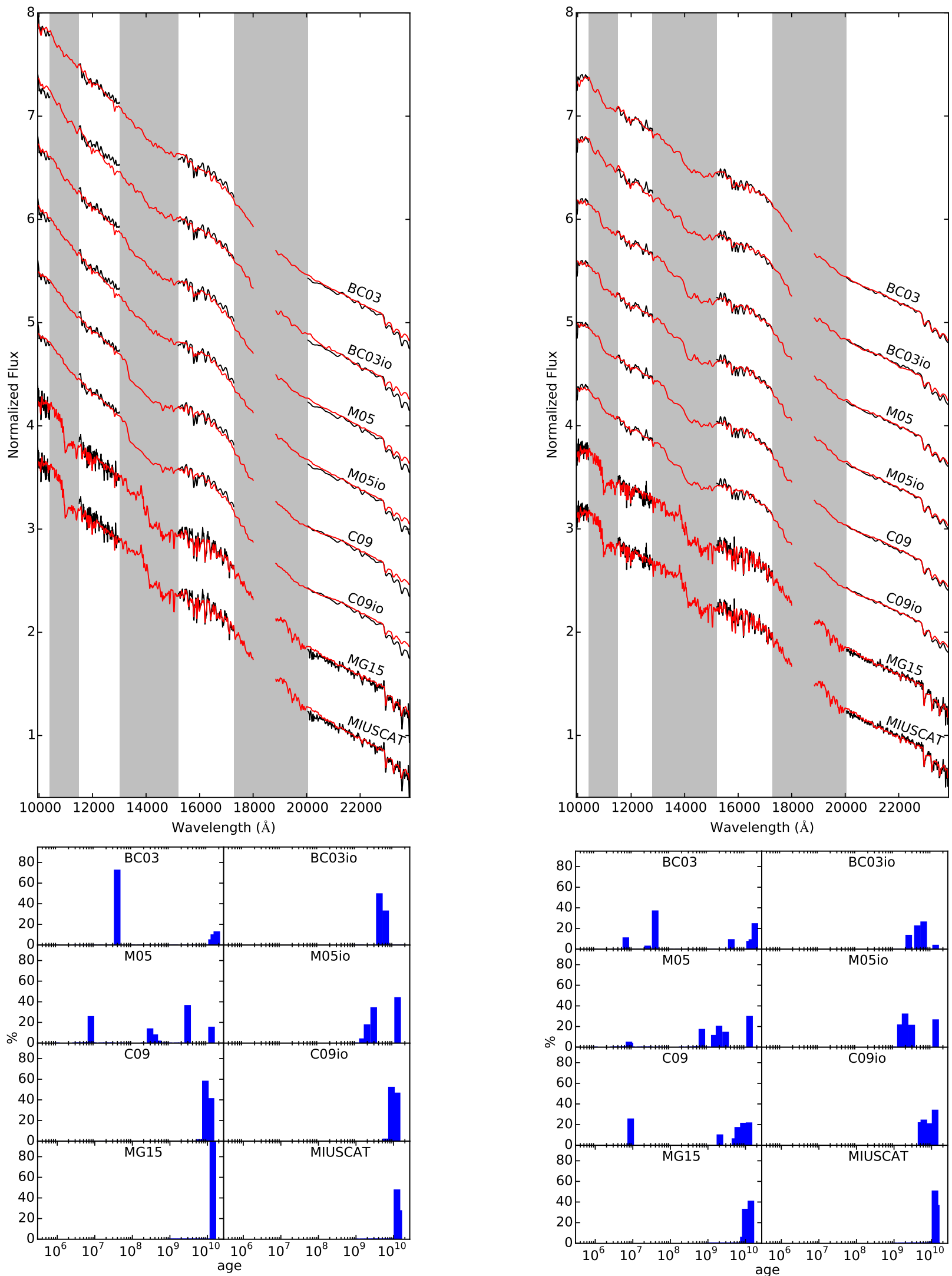

Figure 6. Same as Fig. 4 but for NGC 5966.

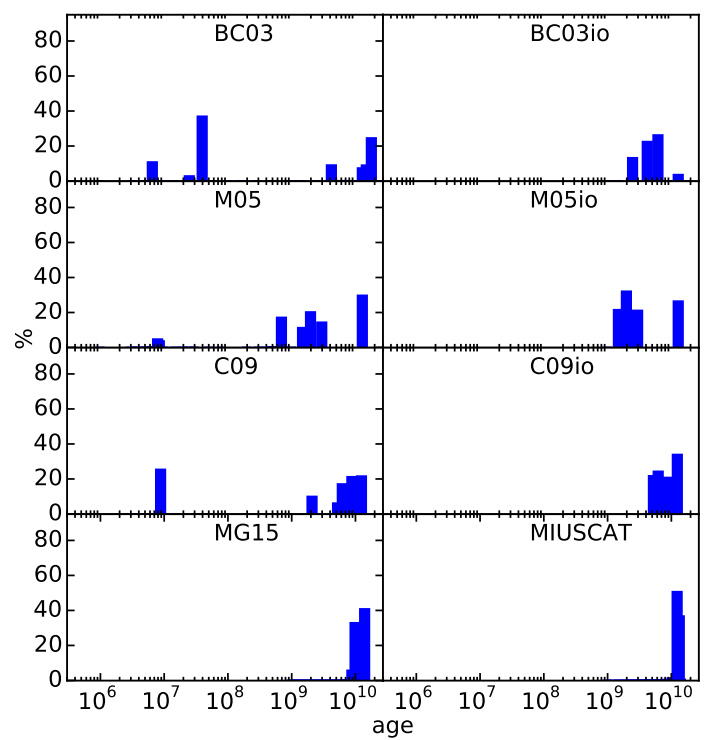

Figure 7. Same as Fig. 4 but for NGC 6081. 

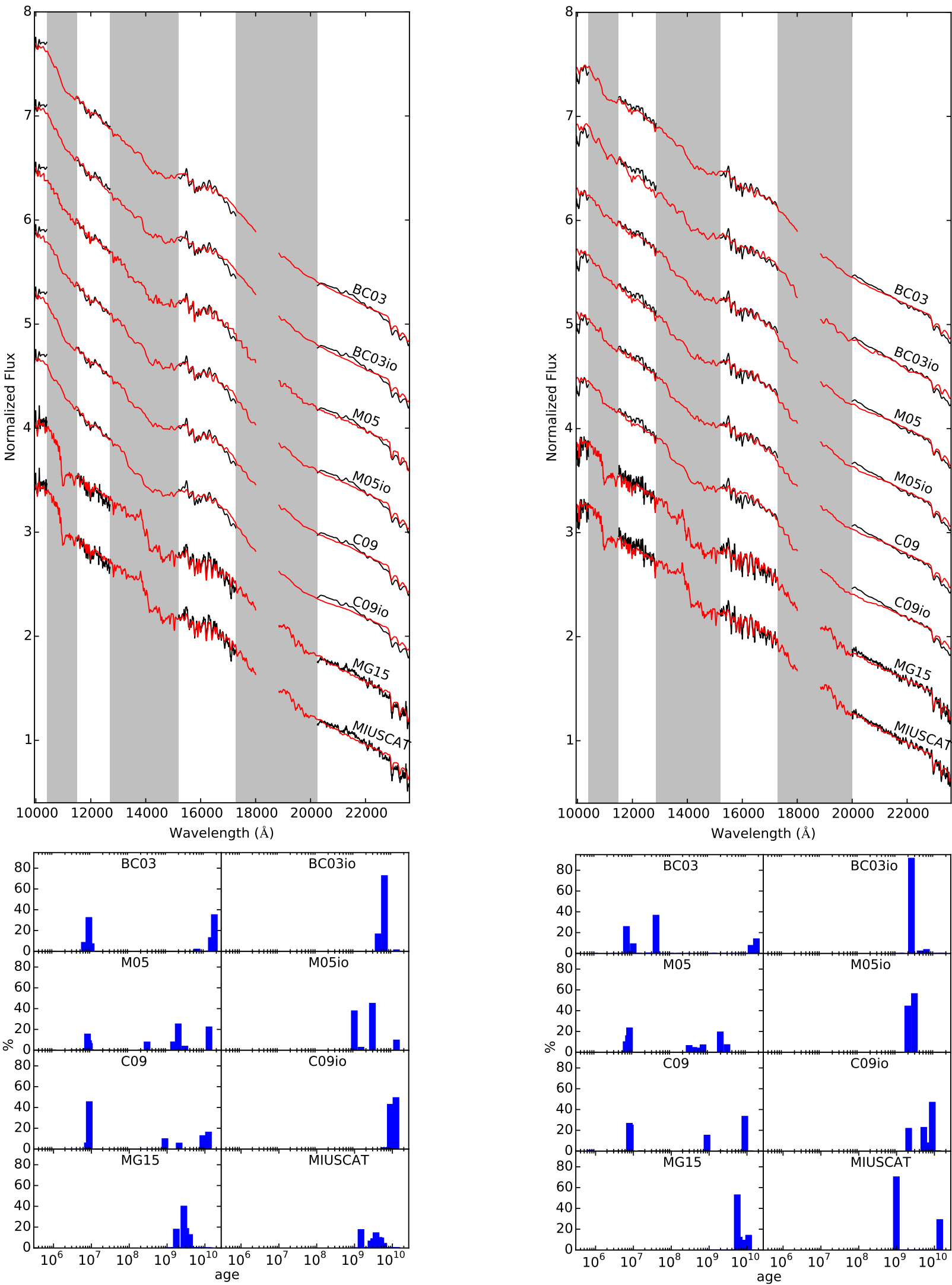

Figure 8. Same as Fig. 4 but for NGC 6146.

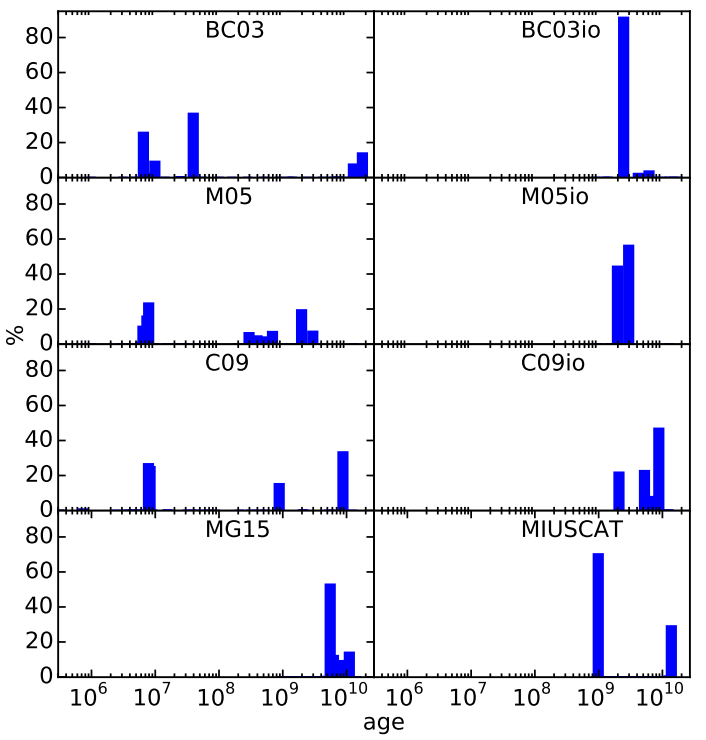

Figure 9. Same as Fig. 4 but for NGC 6338. 

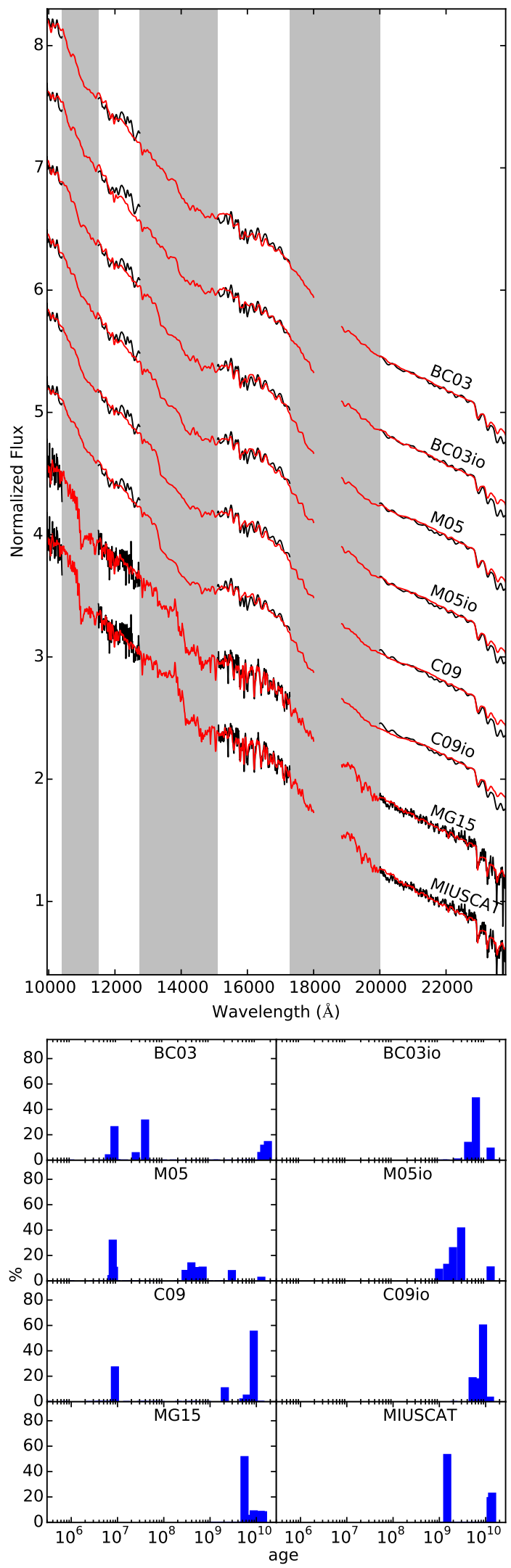

Figure 10. Same as Fig. 4 but for UGC 08234.
Regarding TP-AGB treatment, BC03 uses low-resolution stellar templates from Höfner et al. (2000), while M05 and C09 use higher resolution TP-AGB spectra from Lançon \& Mouhcine (2002), degrading them to match the rest of the spectra. MG15 and MIUSCAT use the IRTF library, which also contains TP-AGB stars (Riffel et al. 2015).

Since our objects may host a low-luminosity AGN (LLAGN), we followed Riffel et al. (2009, and references therein). In addition, we added to the base set a power law with $F_{v} \propto v^{-1.5}$ to represent the featureless continuum (FC) emission from the AGN. Moreover, in the NIR, eight planck distributions with temperatures from 700 to $1400 \mathrm{~K}$ were also added, in steps of $100 \mathrm{~K}$. They represent hot dust continuum heated by the AGN (see Riffel et al. 2009).

\subsection{Fitting procedures}

In order to fit the stellar populations, we used the STARLIGHT code, which is described in Cid Fernandes et al. (2004, 2005). In summary, the code fits the observed spectrum with a combination of SSPs in different proportions. It also searches for the internal extinction that best describes the observed spectrum. To this aim, we used the Cardelli, Clayton \& Mathis (1989) reddening law. Basically, by using a $\chi^{2}$ minimization approach, STARLIGHT fits an observed spectrum $O_{\lambda}$ with a combination of $N_{\star}$ SSPs solving the equation:

$M_{\lambda}=M_{\lambda 0}\left[\sum_{j=1}^{N \star} x_{j} b_{j, \lambda} r_{\lambda}\right] \otimes G\left(v_{\star}, \sigma_{\star}\right)$,

where $M_{\lambda}$ is a model spectrum, $M_{\lambda_{0}}$ is the flux at the normalized point $\lambda_{0}, N_{\star}$ is the number of SSPs used to compose the model, $\vec{x}$ is the population vector so that $x_{j}$ indicates the contribution from the $\mathrm{j}$-esim SSP normalized at $\lambda_{0}, b_{j, \lambda}$ is the $\mathrm{j}$-esim model spectrum, $r_{\lambda}$ is the reddening factor $r_{\lambda}=10^{-0.4\left(A \lambda-A \lambda_{0}\right)}$, which is parametrized by the dust extinction, $A v$. The stellar velocity dispersions and group velocities are modelled by a Gaussian function $G\left(v_{\star}, \sigma_{\star}\right)$.

\section{RESULTS}

The upper panels of Figs 4-10 show, for each galaxy of the sample, the observed (black) and modelled (red) spectra for each of the eight libraries of models employed. Areas with high atmospheric absorption were shaded in the figures. The bottom panels show in blue the luminosity contributions (metallicities summed) for every age of the best model fitted by STARLIGHT.

Since small differences in the ages of the stellar populations are washed away by the noise present in real data, a more consistent and robust way to present the results is in the form of condensed population vectors (Cid Fernandes et al. 2004, 2005). With this in mind, we followed Riffel et al. (2009) and defined the light fraction population vectors as follows: $x y(t \leq 50 \mathrm{Myr})$, $x i(50 \mathrm{Myr}<t \leq 2 \mathrm{Gyr})$ and $x o(t>2 \mathrm{Gyr})$ to represent the young, intermediate-age, and old stellar population vectors, respectively. The same age bins were used for the mass fraction population vectors. Besides, we defined cold and hot dust emission vectors, $B B_{\mathrm{c}}$ for $T \leq 1000 \mathrm{~K}$ and $B B_{\mathrm{h}}$ for $T>1000 \mathrm{~K}$. The results for these binned population vectors for each galaxy are presented in Table 3.

We also followed 'Cid Fernandes et al. (2005), who proposed two additional parameters to describe the SP mixture of a galaxy. These 
Table 3. Results for the NIR synthesis.

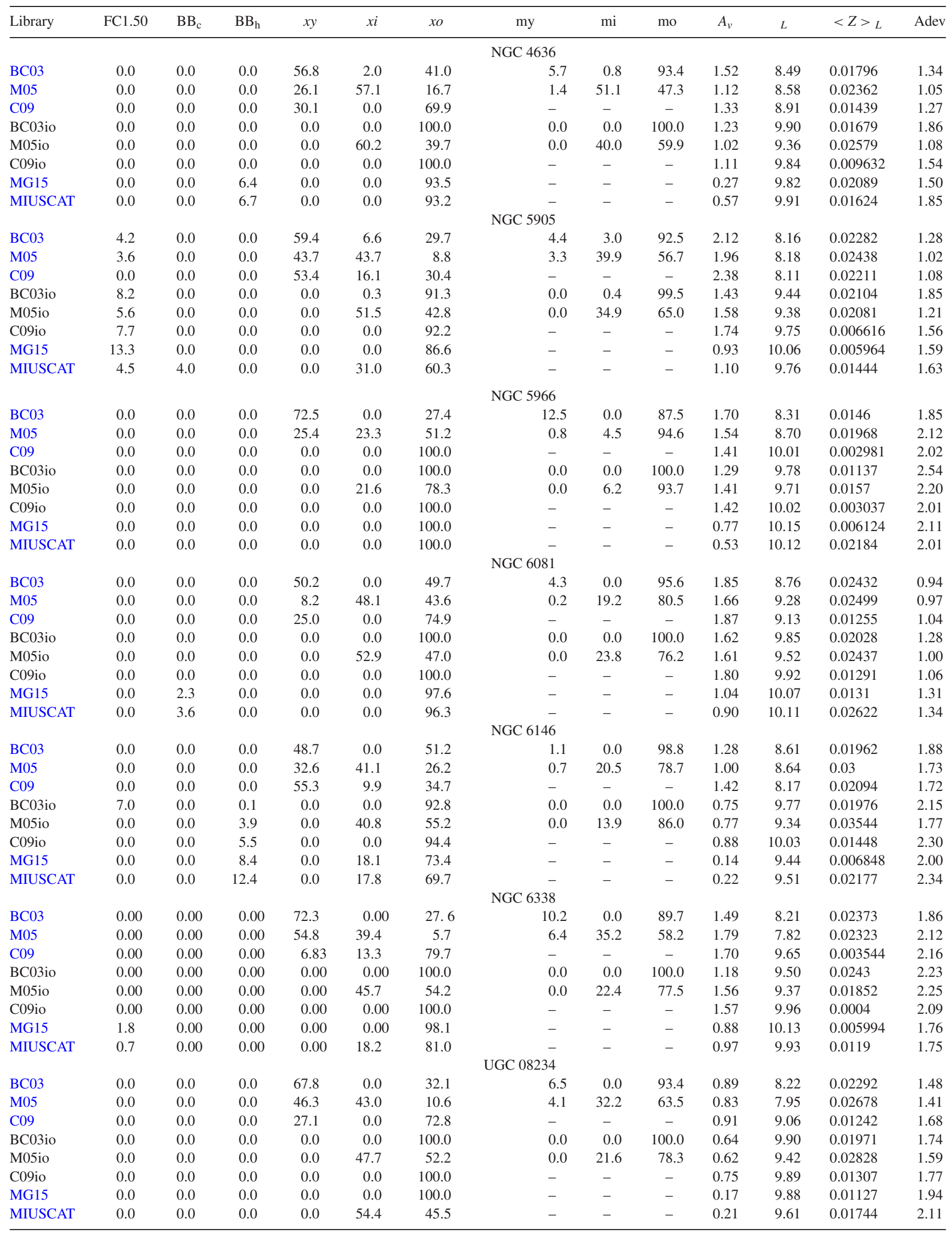




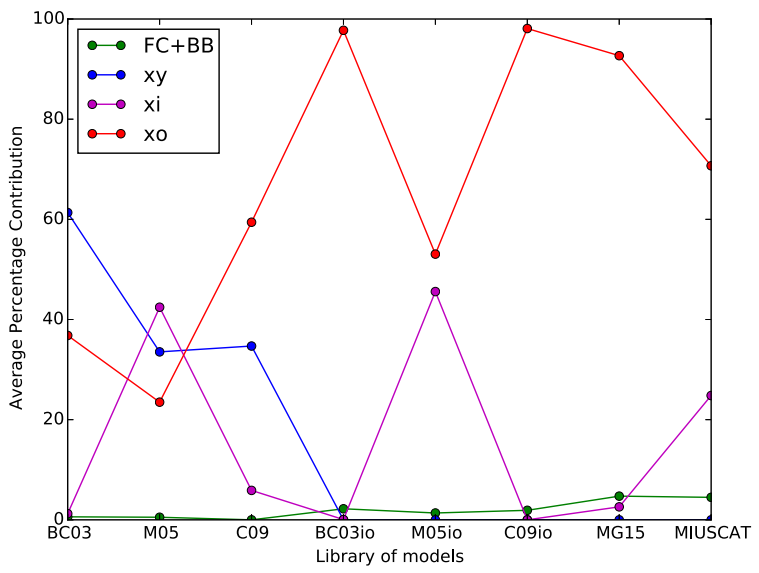

Figure 11. Average percentage contribution for each library of models. Young populations are displayed in blue, intermediate-aged populations in magenta and old stellar populations in red. In green, the summed contributions from featureless continuums and black bodies.

parameters are the mean stellar age $(<t>)$ and mean metallicity $(<Z>)$, which are defined by the following equations:

$$
\begin{aligned}
& <t>_{L}=\sum_{j=1}^{N \star} x_{j} \log \left(t_{j}\right), \\
& <Z>_{L}=\sum_{j=1}^{N \star} x_{j} Z_{j},
\end{aligned}
$$

where $t_{j}$ and $Z_{j}$ are the age and metallicity of the j-esim SSP. The $x_{j}$ percentage contribution can be weighted by light $(L)$ and mass $(M)$ fractions. These parameters, together with the Adev, are also listed in Table 3 (Adev is a value that measures the fit quality, according to the relation $\left|O_{\lambda}-M_{\lambda}\right| / O_{\lambda}$ ). For a better visualization of the trends on each library of models, we summed the percentage contribution of the seven objects and presented them in Fig. 11.

Fits performed with the BC03 library of models display higher contribution from young populations, a mild contribution from old populations, no contribution of intermediate-age populations, and a higher $A_{\mathrm{v}}$ when compared with the other models. With M05, the sample shows a dominance of intermediate-age populations, with significant amounts of young and old populations. When using C09 library, STARLIGHT finds a dominance of old stellar populations, with mild contribution from young populations. When using lowresolution models that do not include young populations, BC03io and C09io models result in an $\sim 97$ per cent contribution from old populations for all the objects, with the other 3 per cent due to the FC+BB. When using M05io models, on the other hand, three objects (NGC 4636, NGC 5905, and NGC 6081) appear dominated by intermediate-age populations, with old populations dominating the rest of the sample. With high-spectral-resolution libraries of models, a dominance of old populations was found, with higher contributions from $\mathrm{FC}$ and $\mathrm{BB}$ when compared with lower resolution ones. However, results obtained using MIUSCAT library display a trend towards intermediate-age SSPs whereas results obtained using MG15 library display a trend towards old SSPs. For NGC 6338 and UGC 08234, STARLIGHT found intermediate-age populations dominating the emission ( 70 per cent and 54 per cent, respectively) when

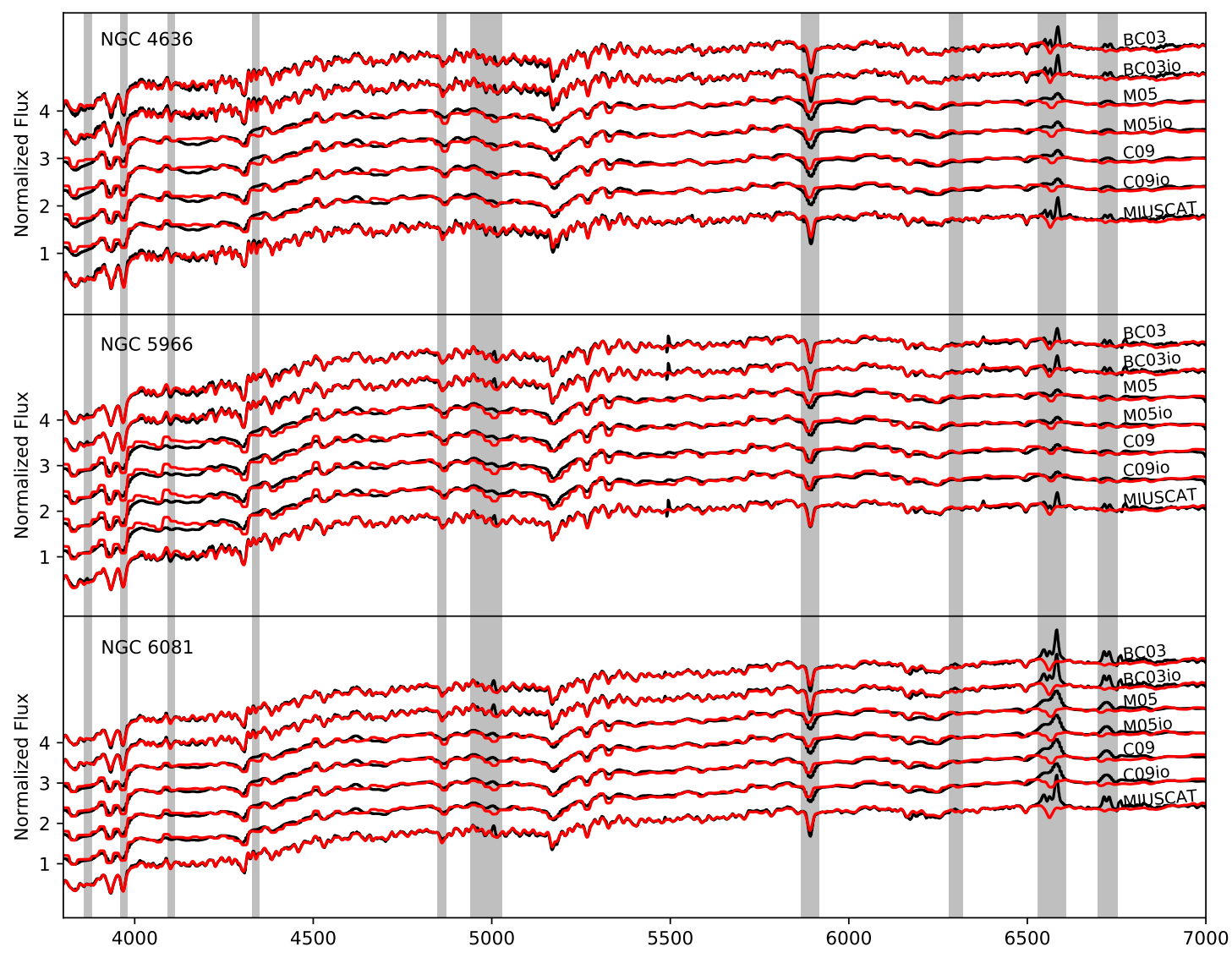

Figure 12. Optical fits for NGC 4636, NGC 5966, and NGC 6081. For each galaxy, we present the observed (black) and modelled spectra (red) for the four libraries used. 


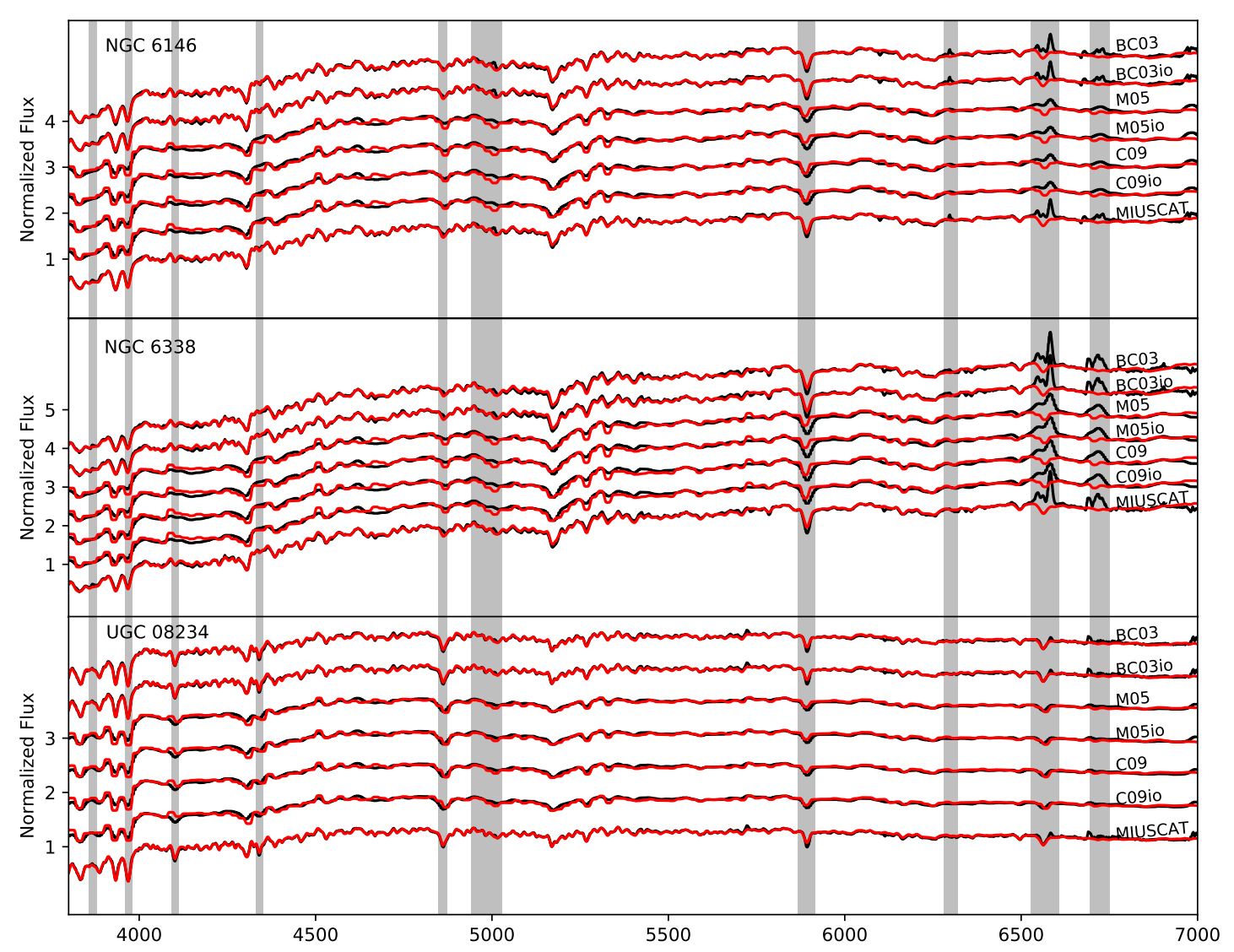

Figure 13. Same of Fig. 12 but for NGC 6146, NGC 6338, and UGC 08234.

using MIUSCAT library, while no contribution was found when using MG15. For NGC 5905, a contribution of 31 per cent from an intermediate-age population was found when using MIUSCAT library, while no contribution was found for this age when using MG15. For a better visualization of these differences, we plotted the average contribution from young, intermediate-age, and old populations plus the summed contributions of the FC and BB in Fig. 11.

The stellar synthesis using low-spectral-resolution libraries of models did not fit properly the $2.3 \mu \mathrm{m}$ CO bands on NGC 5966, NGC 6146, NGC 6338, and UGC 08234 regardless of the lowresolution library of models used. The $\mathrm{Na}$ I and $\mathrm{Ca}$ I lines in the $K$ band and the absorptions in the $J$ and $H$ band were not fitted with any low-resolution library. A similar result was reported by Riffel et al. (2015), who found that none of the models tested by them (BC03; M05; Maraston \& Strömbäck 2011) accurately reproduces all of the stellar features observed in the spectra. In all cases, the fits improved when young populations were included. When using high-resolution libraries, the absorption features in $H$ and $K$ bands were fitted with a much better agreement between models and observations. However, in the $J$ band, because of the low $\mathrm{S} / \mathrm{N}$, the fits are not as good. Note that this is not a big issue because this band is mostly used for fitting the continuum inclination.

\section{DISCUSSION}

\subsection{NIR models with low spectral resolution}

Our sample is composed mainly of ETGs (NGC 4636, NGC 5966, NGC 6081 NGC 6146, NGC 6338, and UGC 08234), which are mainly constituted of old populations (Rickes et al. 2009, and references therein). Analysing Table 3, it is clear that for libraries of models with low spectral resolution (BC03; M05; and C09), the results are linked to the library used rather than to the galaxy properties themselves. This result is different from the optical result reported by Chen et al. (2010). They found that changing the library would result in a change of the percentage contributing light fractions, even though the dominant populations are unaltered. They also compared high- and low-resolution libraries, and the dominant populations still remained unchanged. This is not the case for the NIR. We found from Figs 4-10 (see also Table 3) that for the NIR spectral range, the dominant population is highly dependent on the chosen models.

According to our results, $\mathrm{BC} 03$ models give the highest contribution from young populations in the whole sample. The higher young SP contributions are also linked to a higher reddening value, as we can see from Table 3. This result is in agreement with those found by Riffel et al. (2015). However, they are in contrast with the ones reported by Capozzi et al. (e.g. 2016), who found that the inclusion of TP-AGB stars tends to produce results with older ages. This difference might be connected to the fact that they used panchromatic spectral energy distributions, while we used only NIR spectra to make the comparison.

Indeed, for models with low spectral resolution, the large variation in age when young populations are not included implies that the synthesis is unable to distinguish between an old population and a reddened younger one or a more metallic one, since in all cases, the younger populations are followed by a higher extinction or a higher metallicity. This result was first discussed by Worthey (1994) and seems to still hold in the NIR. 

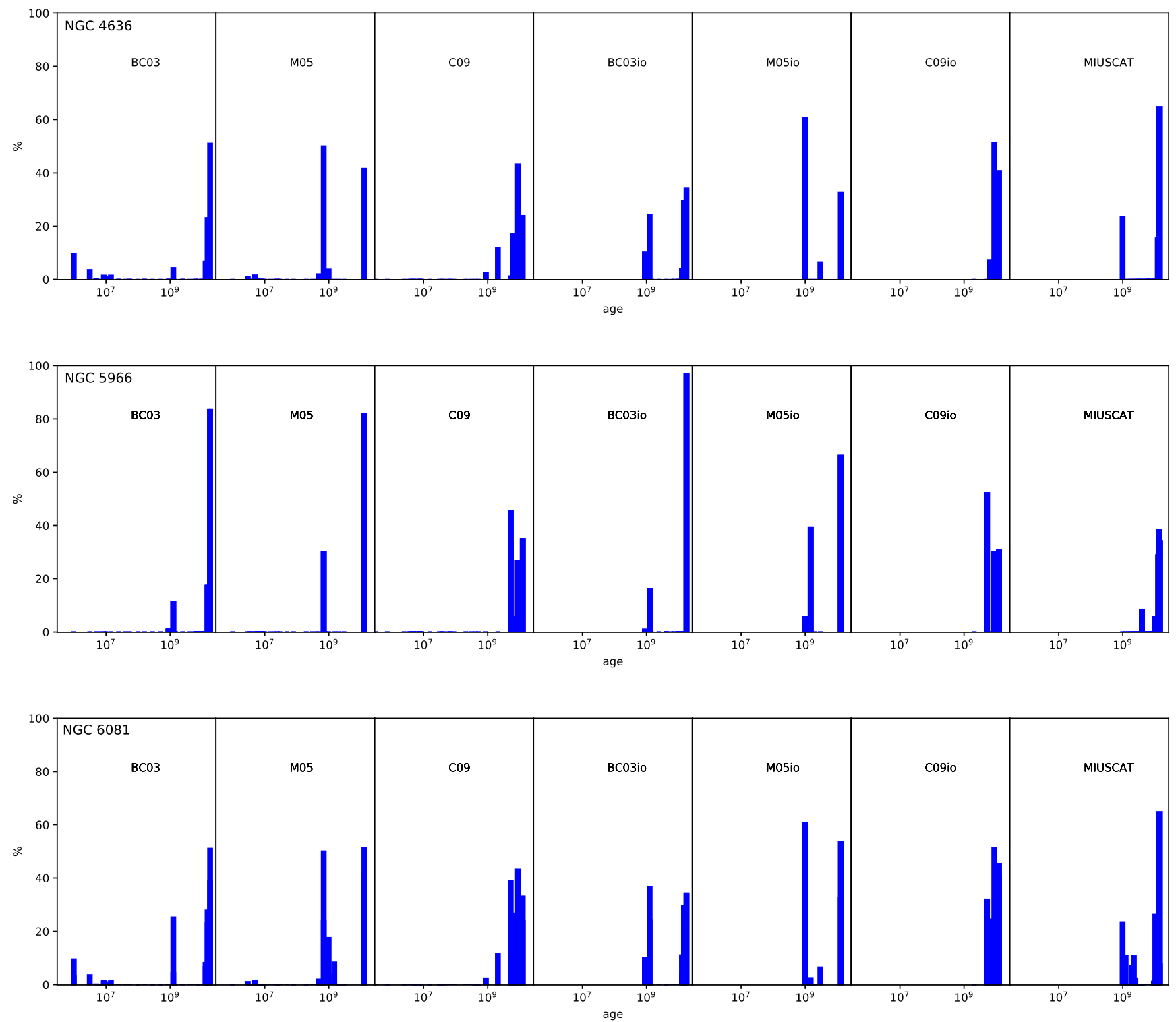

Figure 14. Optical star formation histories for NGC 4636, NGC 5966, and NGC 6081, metallicities summed.

Regarding the metallicity, when young populations are present in the fitting process, both $\mathrm{BC} 03$ and M05 find values close to solar. $\mathrm{C} 09$, on the other hand, tends to find subsolar values of metallicity. When young ages are removed, the same results still hold. Concerning the high-resolution libraries, MIUSCAT also finds values close to $\mathrm{Z}_{\odot}$, whereas MG15 tends to find subsolar values.

Also, all model sets with low spectral resolution found high $\left(A_{\mathrm{v}} \gtrsim 1.0 \mathrm{mag}\right.$ ) values of extinction. These results are uncompatible with literature results which show that ETGs have negligible amount of dust (Padilla \& Strauss 2008).

\subsection{NIR models with high spectral resolution}

For EPS models with high spectral resolution (MG15 and MIUSCAT), the above scenario improved considerably. For three objects (NGC 4636; NGC 5966; and NGC 6081), besides the small ( $<10$ per cent) contribution from dust, only old SSPs were found using both libraries of models. For NGC 6146, both libraries predict a contribution of intermediate-age populations of $\sim 18$ per cent.
For NGC 5905, the only spiral galaxy in our sample, a contribution of 31 per cent from intermediate-age stars was obtained with MIUSCAT, but this same percentage was not detected when using MG15. Lastly, for UGC 08234, the fit with MG15 SSPs appears dominated by old populations. However, when MIUSCAT models are employed, the fits become dominated by intermediate-age stellar populations.

Note that it is not possible to fully test libraries with high spectral resolution, because MG15 and MIUSCAT are only composed of SSPs older than 1 Gyr. However, the results we obtained point towards an improvement when using libraries with high resolution. Also, the dust extinction values found with high-spectral-resolution libraries are much more consistent with literature results that showed that ETGs have very low amounts of dust.

\subsection{Comparison with optical results}

For the optical region, we also fit the data using the different libraries of models. Since MG15 models are only available in the 

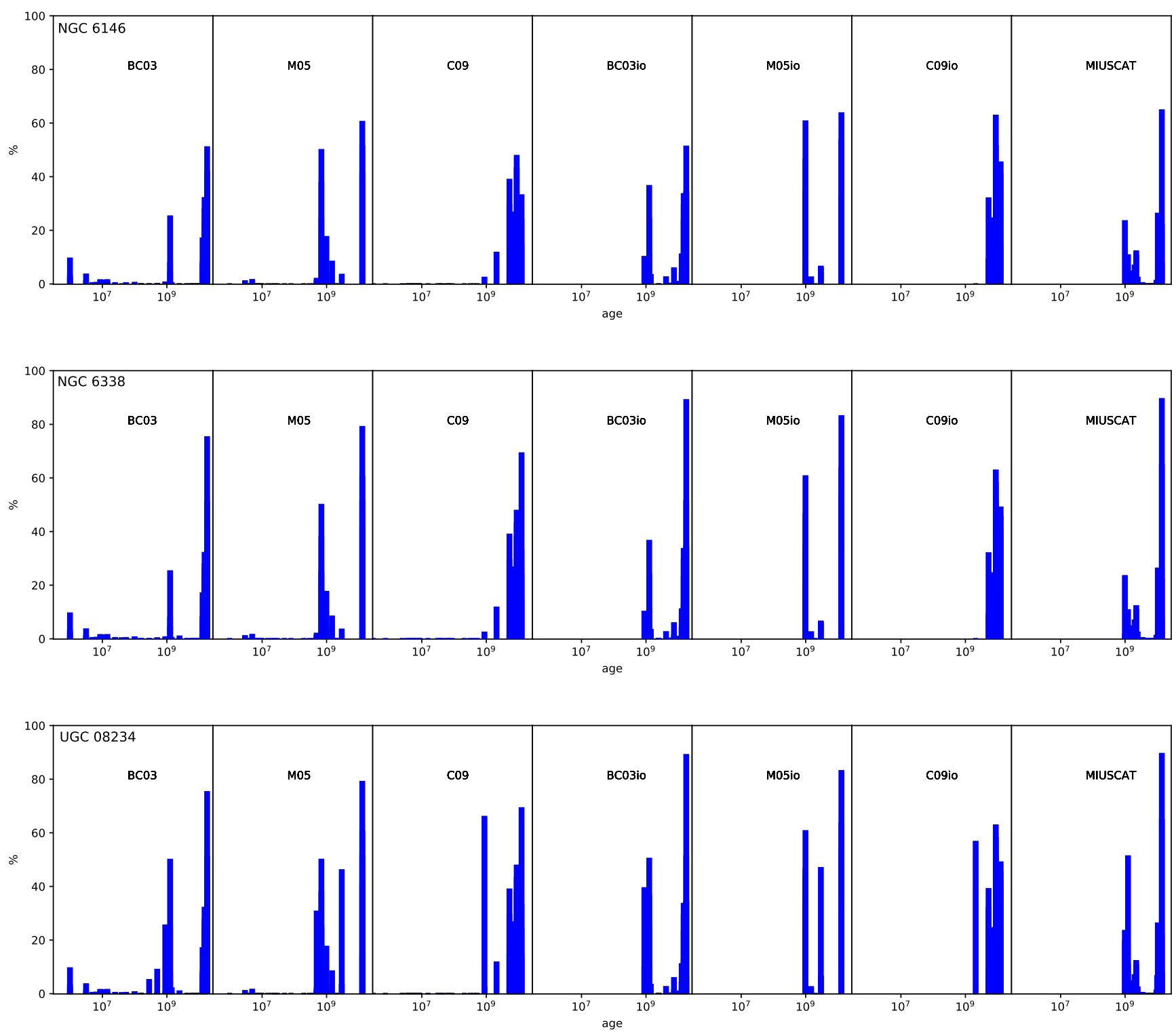

Figure 15. Same as Fig. 14 but for NGC 6146, NGC 6338, and UGC 08234.

NIR, we performed the synthesis using only BC03, M05, C09, and MIUSCAT libraries. Also, for a fair comparison between optical and NIR results, we performed the fits using the libraries without the young ages, i.e. BC03io, M05io, and C09io. Figs 12 and 13 present the optical synthesis results for six galaxies with optical spectra. For each galaxy, we show the observed spectra in black and the model spectrum in red. On Figs 14 and 15, we show the light fraction for each age in blue. The binned light and mass contribution, along with the $A_{\mathrm{v}},{ }_{L},<Z>{ }_{L}$ and the Adev for each galaxy, are shown in Table 4.

In the optical synthesis, the different libraries of models produced results that are more similar among each other than those derived from the NIR. The only exception was the fits with M05 and M05io models, that also in the optical found a high fraction of intermediate-age populations. For the other libraries, in the optical region, the synthesis found a dominant contribution of old populations to the light of NGC 4636, NGC 5966, NGC 6081, NGC 6146, and NGC 6338 and a dominance of intermediate-age populations for UGC 08234 . Using M05, on the other hand, the synthesis found similar results for all the objects, with small ( $\lesssim 5$ per cent $)$ contributions of young populations and higher fractions of intermediate-age ( 25 percent $\lesssim x i \lesssim 55$ percent) and old (40 per cent $\lesssim x i \lesssim 75$ per cent) populations. It is worth mentioning that when using BC03 to fit the optical spectra, a larger contribution from young stellar populations is found compared to the other libraries. However, the dominant old population (or intermediate for UGC 08234) still remains the same found with the other libraries (see Table 4), while in the NIR spectral range, the dominant population contributing to the galaxy emission was different (see Table 3). Removing the SSPs with $t<1 \mathrm{Gyr}$ from BC03 did not change the results.

For a proper comparison between optical and NIR results, we present in Figs 16-23 the values of $x y$, xi, xo, $A_{\mathrm{v}},{ }_{L}$, and $\left\langle Z>_{L}\right.$ found by STARLIGHT both for the optical and NIR ranges. The $x$ axis displays the optical results and the $y$-axis displays the NIR results found by STARLIGHT for eight different libraries of models used. For MG15 library, since it only has the NIR range of the spectra, we compared it to the optical results obtained with 
Table 4. Results for the optical synthesis.

\begin{tabular}{|c|c|c|c|c|c|c|c|c|c|c|}
\hline Library & $x y$ & $x i$ & xo & my & $\mathrm{mi}$ & mo & $A_{\mathrm{v}}$ & $L$ & $<Z>_{L}$ & Adev \\
\hline $\mathrm{BC} 03$ & 17.1 & 4.3 & 78.4 & 0.0 & 0.1 & 99.8 & -0.01 & 9.51 & 0.02398 & 1.93 \\
\hline C09 & 0.0 & 2.4 & 97.5 & - & - & - & 0.25 & 9.86 & 0.01811 & 2.37 \\
\hline BC03io & 0.0 & 33.7 & 66.2 & 0.0 & 1.2 & 98.8 & 0.02 & 9.82 & 0.02305 & 2.02 \\
\hline M05io & 0.0 & 60.7 & 39.2 & 0.0 & 14.2 & 85.7 & 0.37 & 9.39 & 0.0296 & 2.59 \\
\hline \multicolumn{11}{|c|}{ NGC 5966} \\
\hline $\mathrm{BC} 03$ & 0.0 & 11.0 & 88.4 & 0.0 & 1.0 & 98.9 & -0.01 & 10.11 & 0.02526 & 1.84 \\
\hline M05 & 0.0 & 26.8 & 73.2 & 0.0 & 2.5 & 97.4 & 0.26 & 9.77 & 0.02563 & 3.61 \\
\hline C09 & 0.0 & 0.0 & 100.0 & - & - & - & 0.16 & 9.90 & 0.02232 & 3.89 \\
\hline BC03io & 0.0 & 15.1 & 84.8 & 0.0 & 1.4 & 98.5 & -0.00 & 10.08 & 0.0265 & 1.83 \\
\hline M05io & 0.0 & 40.5 & 59.5 & 0.0 & 9.9 & 90.0 & 0.23 & 9.73 & 0.02708 & 3.65 \\
\hline M05 & 0.0 & 49.8 & 50.1 & 0.0 & 6.5 & 93.4 & 0.91 & 9.53 & 0.02232 & 2.48 \\
\hline $\mathrm{C} 09$ & 0.0 & 0.0 & 100.0 & - & - & - & 0.85 & 9.87 & 0.01503 & 2.60 \\
\hline ВC03io & 0.0 & 34.6 & 65.3 & 0.0 & 1.6 & 98.3 & 0.71 & 9.82 & 0.01778 & 1.69 \\
\hline M05io & 0.0 & 47.7 & 52.2 & 0.0 & 6.1 & 93.8 & 0.88 & 9.59 & 0.0181 & 2.51 \\
\hline C09io & 0.0 & 0.0 & 100.0 & - & - & - & 0.84 & 9.92 & 0.01447 & 2.59 \\
\hline MIUSCAT & 0.0 & 16.7 & 83.2 & - & - & - & 0.92 & 9.83 & 0.02177 & 1.49 \\
\hline \multicolumn{11}{|c|}{ NGC 6146} \\
\hline ВC03 & 6.6 & 9.6 & 83.0 & 0.0 & 0.3 & 99.6 & -0.03 & 9.84 & 0.0213 & 1.29 \\
\hline M05 & 0.0 & 38.6 & 61.4 & 0.0 & 4.4 & 95.5 & 0.28 & 9.60 & 0.02732 & 2.65 \\
\hline C09 & 0.0 & 0.0 & 100.0 & - & - & - & 0.16 & 9.89 & 0.02022 & 2.72 \\
\hline BC03io & 0.0 & 13.3 & 86.6 & 0.0 & 0.5 & 99.5 & -0.02 & 10.04 & 0.01982 & 1.33 \\
\hline M05io & 0.0 & 33.3 & 66.6 & 0.0 & 4.5 & 95.4 & 0.26 & 9.71 & 0.02072 & 2.70 \\
\hline M05io & 0.0 & 21.5 & 78.4 & 0.0 & 2.0 & 97.9 & 0.65 & 9.87 & 0.02038 & 3.22 \\
\hline C09io & 0.0 & 0.0 & 100.0 & - & - & - & 0.53 & 10.03 & 0.01938 & 3.34 \\
\hline MIUSCAT & 0.0 & 3.7 & 96.2 & - & - & - & 0.59 & 10.10 & 0.02628 & 1.63 \\
\hline \multicolumn{11}{|c|}{ UGC 08234} \\
\hline $\mathrm{BC} 03$ & 0.0 & 86.5 & 13.4 & 0.0 & 26.2 & 73.7 & -0.07 & 9.15 & 0.04017 & 1.18 \\
\hline M05 & 0.0 & 40.0 & 60.0 & 0.0 & 8.5 & 91.4 & 0.03 & 9.28 & 0.02446 & 2.12 \\
\hline C09 & 0.0 & 65.5 & 34.4 & - & - & - & 0.23 & 9.28 & 0.02118 & 2.32 \\
\hline BC03io & 0.0 & 85.0 & 14.9 & 0.0 & 25.9 & 74.1 & -0.07 & 9.21 & 0.0368 & 1.18 \\
\hline M05io & 0.0 & 42.7 & 57.2 & 0.0 & 14.0 & 85.9 & -0.05 & 9.34 & 0.01896 & 2.20 \\
\hline C09io & 0.0 & 61.5 & 38.4 & - & - & - & 0.21 & 9.29 & 0.01998 & 2.32 \\
\hline MIUSCAT & 0.0 & 67.1 & 32.8 & - & - & - & 0.07 & 9.40 & 0.02134 & 1.00 \\
\hline
\end{tabular}

MIUSCAT. Over six panels of each picture, we plotted a solid line, representing a perfect correlation between optical and NIR. Over the $x y, x i$, and $x o$ panels, we also plotted two dashed lines showing the 10 percent error margin and two dotted lines showing the 30 per cent error margin. Over the $A_{\mathrm{v}}$ panel, we plotted two dashed lines showing the $0.1 \mathrm{mag}$ error margin and two dotted lines showing the $0.3 \mathrm{mag}$ error margin. For $\langle t\rangle_{L}$ and $\langle Z\rangle_{L}$ panels, since they are logarithmic scales, we did not display error margins.

From Figs 16 to 23, it is possible to see that libraries with low spectral resolution and with young populations usually disagrees in the optical and the NIR about the fractions of each stellar populations that contributes to the galaxy-integrated light. It is clear that the fits using $\mathrm{BC} 03$, BC03io, $\mathrm{C09}$, and C09io overestimate the amount of younger stellar populations in the NIR when compared to the fits in the optical. On the other hand, the fits with M05 and M05io are more self-consistent when comparing optical and NIR results, where in both cases sizable amounts of intermediate-age stars are found. All the tests with the lower spectral resolution models displayed a tendency to find extinctions $\sim 1$ mag higher when fitting the NIR spectral range, compared to optical one. Regarding the metallicity, fits with C09 find lower metallicities in the NIR 

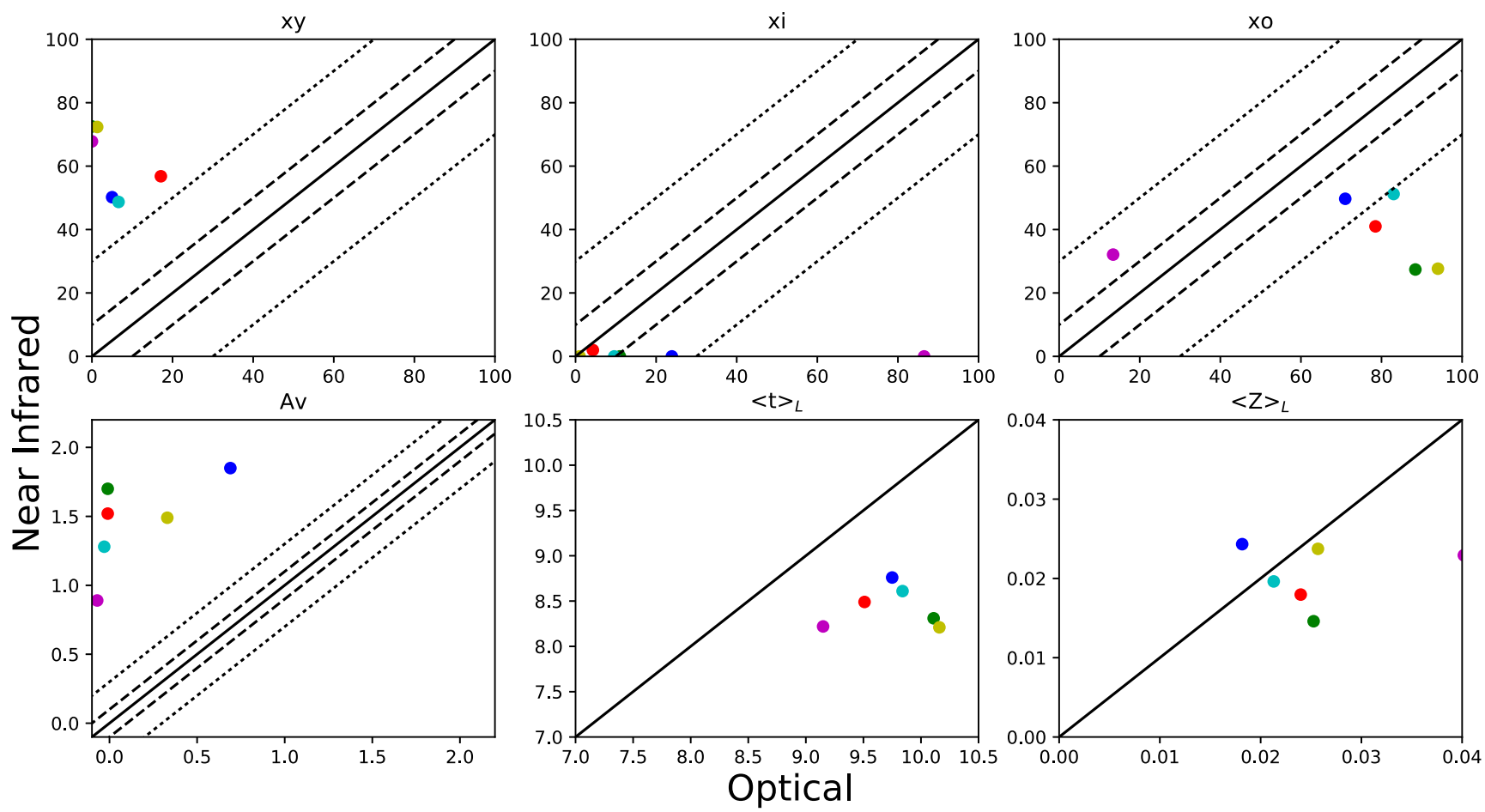

Figure 16. Comparison between optical and NIR results found using BC03 library of models. In the upper panels, we present from left to right the $x y$, $x i$, and $x o$ results. In the bottom panels, we show the $\mathrm{A}_{\mathrm{v}},{ }_{L}$, and $\langle Z\rangle_{L}$ results. Over all panels, we plotted a solid line, representing a perfect correlation between optical and NIR. Also, we plotted dashed and dotted lines representing error margins of 10 and 30 percent in the $x y, x i$, and $x o$ panel and error margins of 0.1 and 0.3 mag in the $A_{\mathrm{V}}$ panel. Each galaxy was plotted in a different colour: NGC 4636, red; NGC 5966, green; NGC 6081, blue; NGC 6146, cyan; NGC 6338, yellow; UGC et al., 8234, magenta.
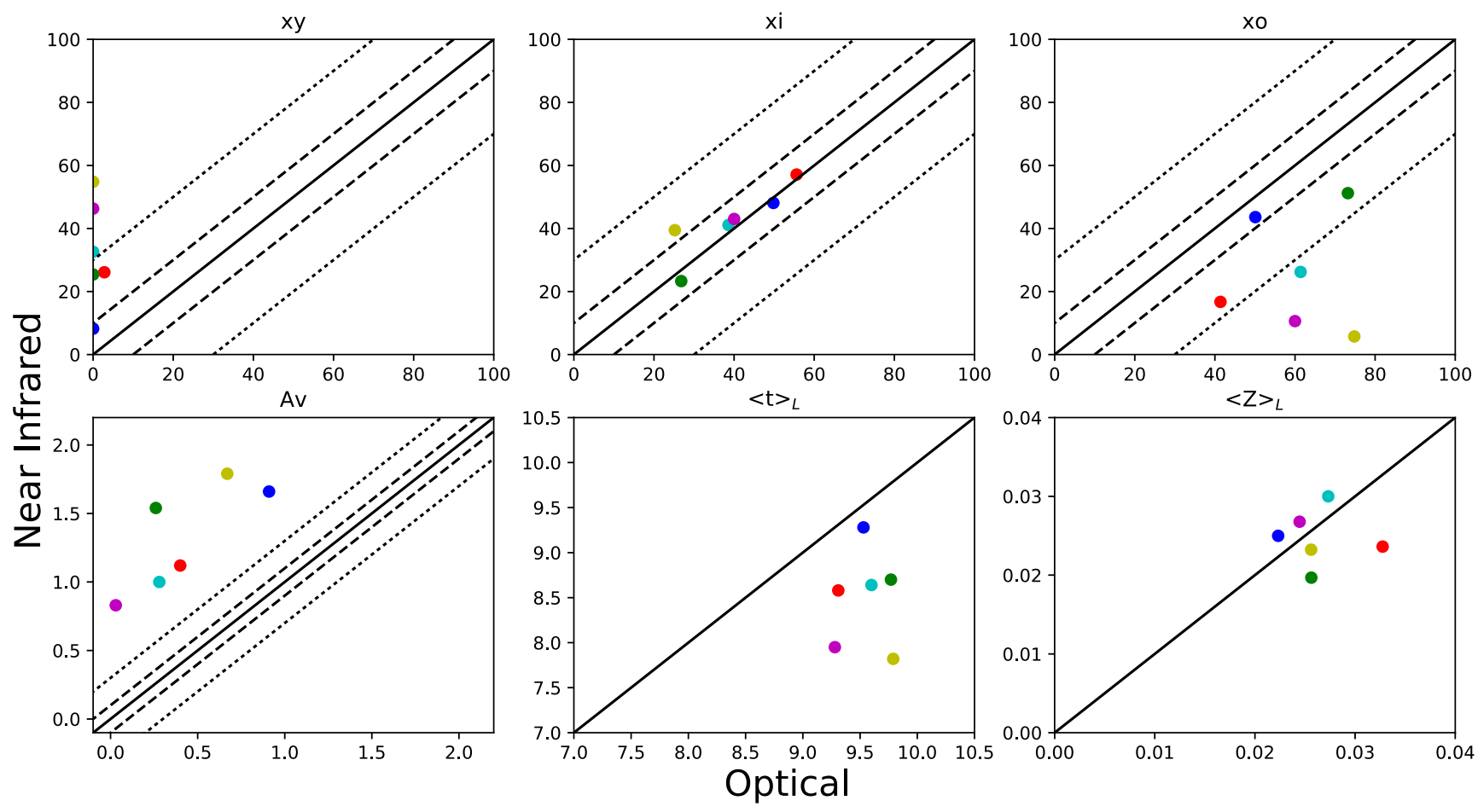

Figure 17. Same of Fig. 16, but for M05 library of models. 

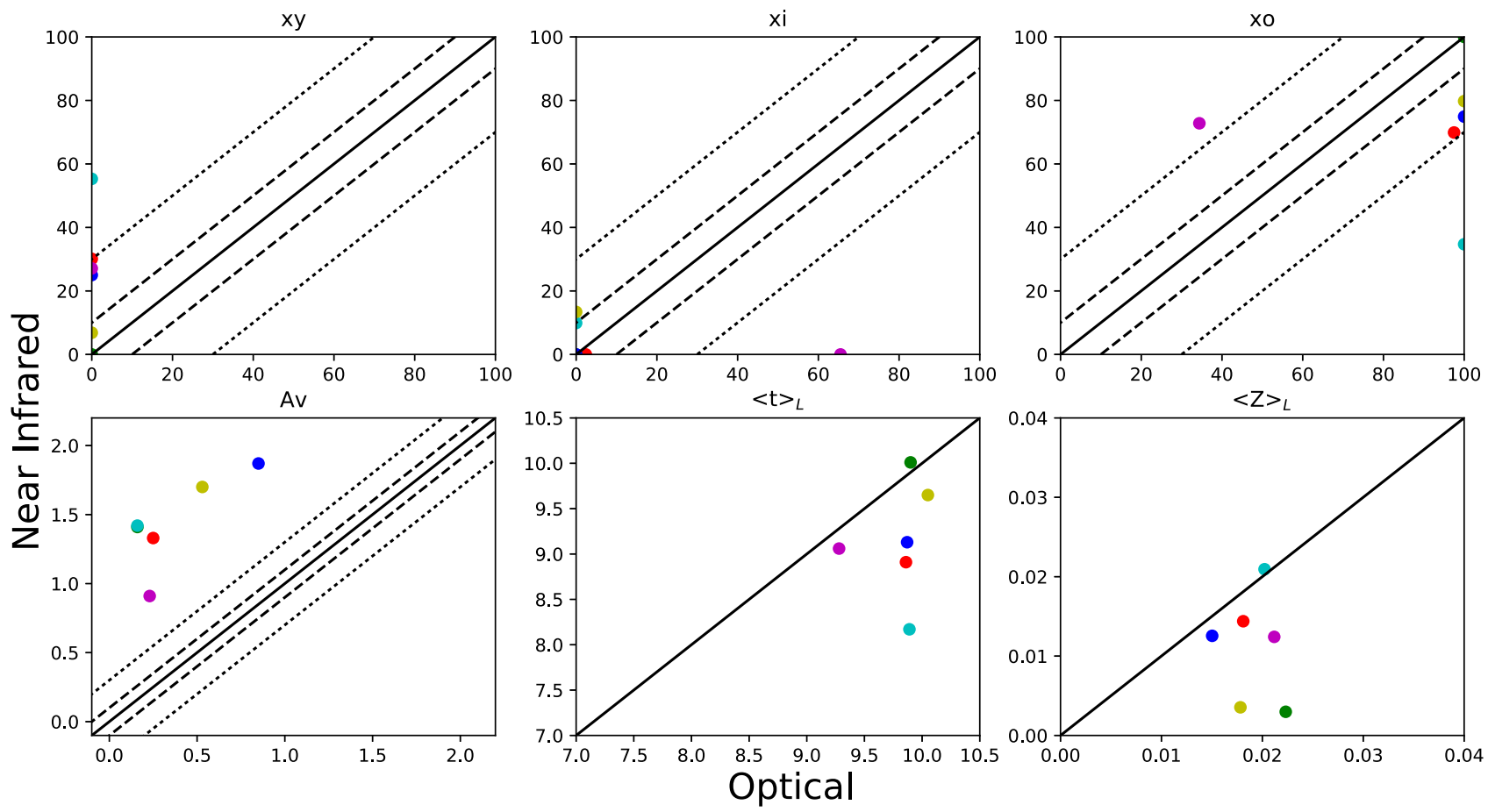

Figure 18. Same of Fig. 16, but for C09 library of models.
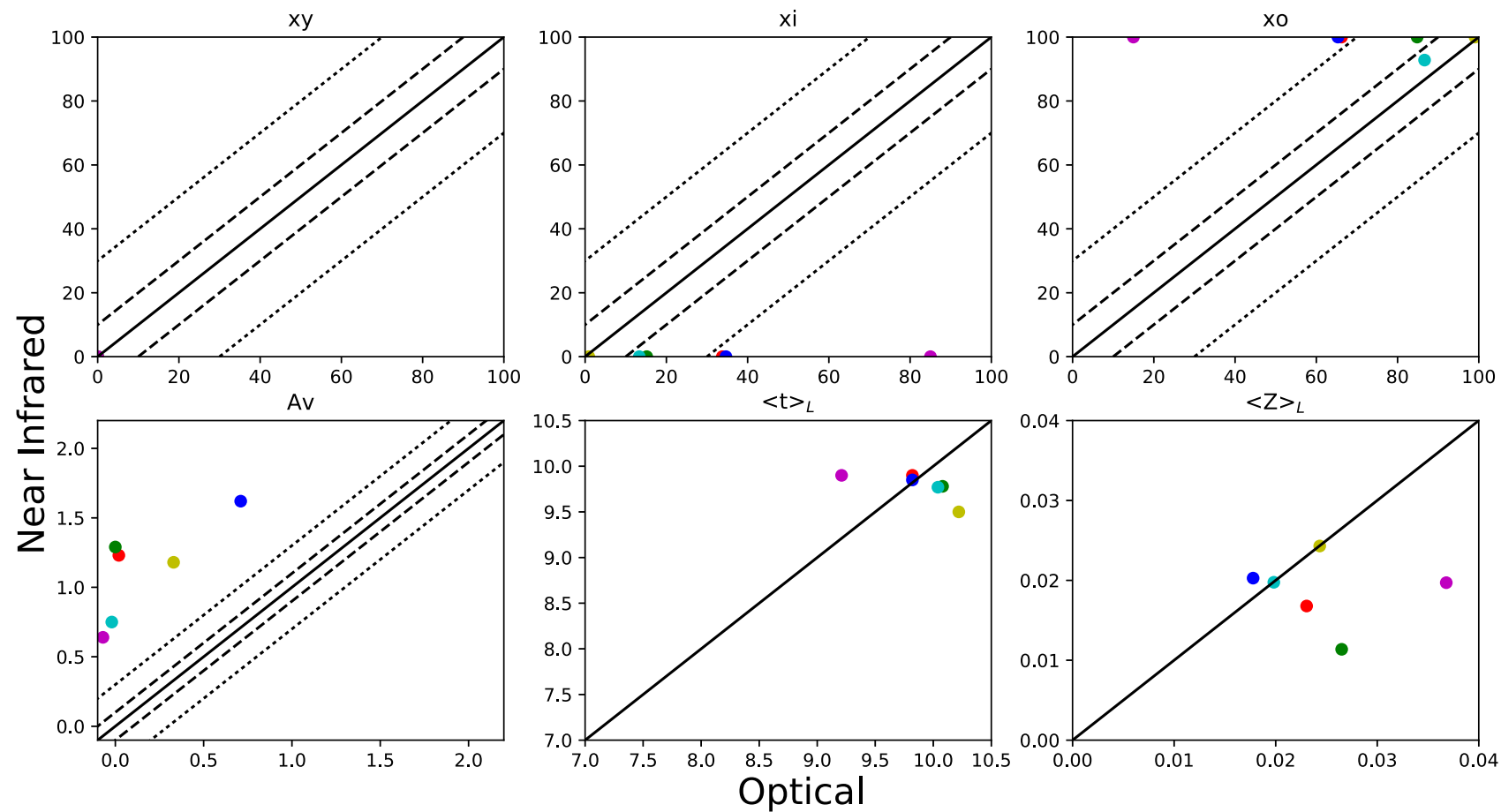

Figure 19. Same of Fig. 16, but for BC03io library of models.

compared to the optical, whereas BC03 and M05 fits using NIR or optical data converge to nearly the same metallicities.

When SSPs with $t \leq 1$ Gyr were removed from the libraries, the stellar populations found in the optical and NIR were much closer to each other. From the three libraries with low-resolution and without young SSPs, M05io was the one that found the most consistent results between the two wavelength ranges, with all the points in the $x i$ and $x o$ panels inside the 30 per cent error margin. However, since the amounts of intermediate-age SSPs found with this library are close to $\sim 50$ per cent and the sample is mainly composed of ETGs, these results suggest that both wavelength ranges tend equally to overestimate the amount of intermediate-age SSPs.

For BC03io, we found larger amounts of intermediate-age stars in the optical than in the NIR, where only old SSPs contributed to the fits. For the tests with C09io, we found a dominant contribution from old SSPs in both spectral ranges. The only exception was 

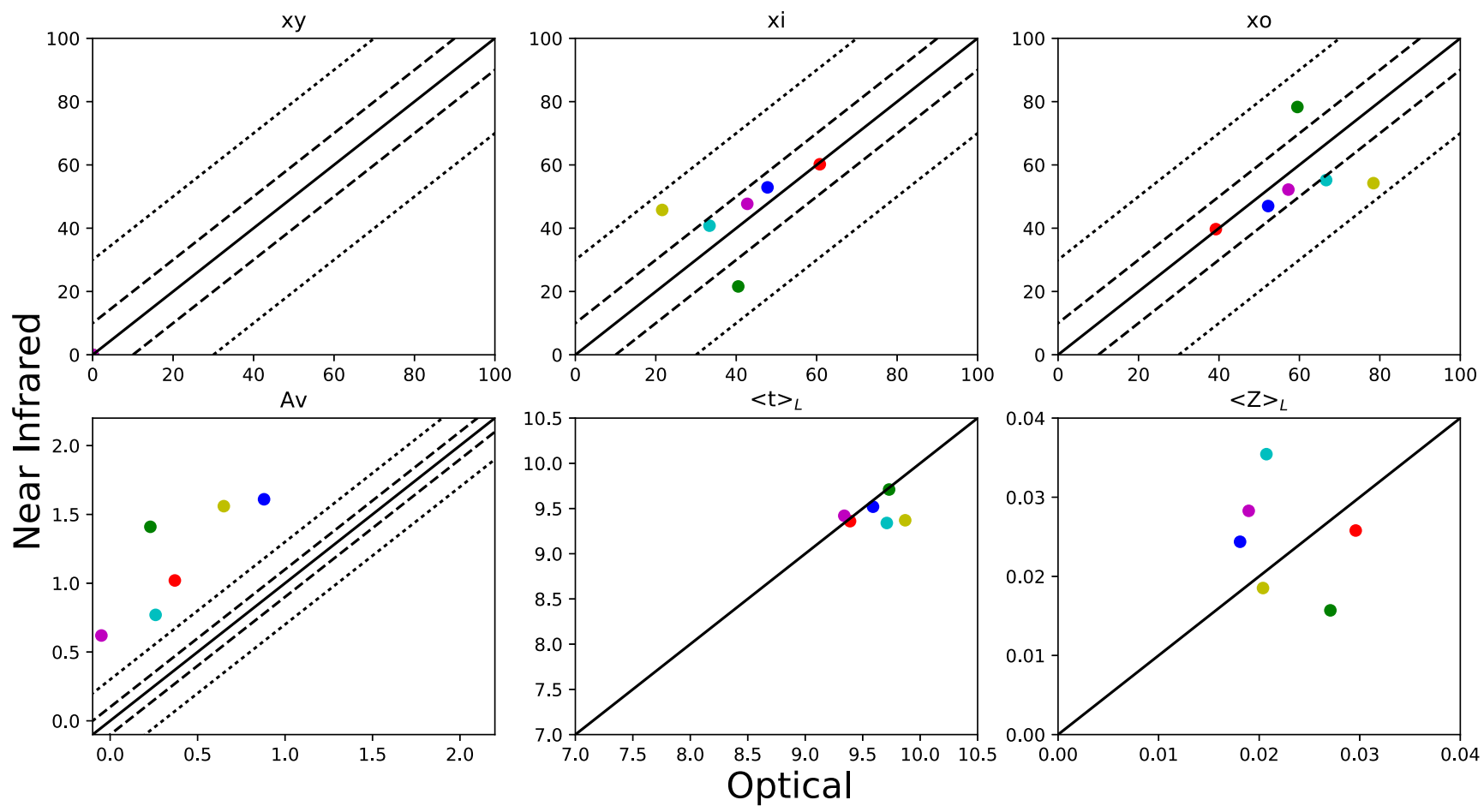

Figure 20. Same of Fig. 16, but for M05io library of models.
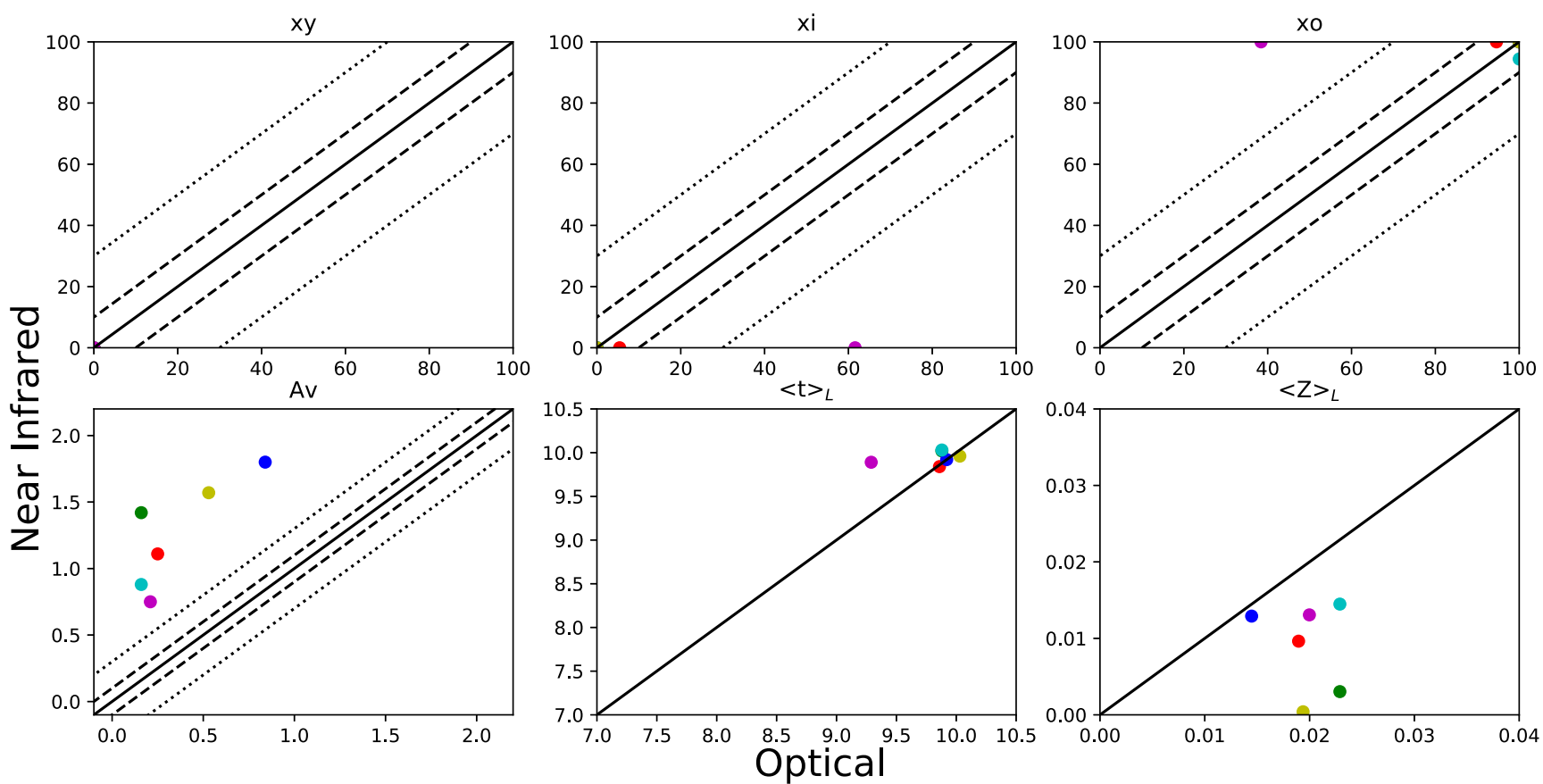

Figure 21. Same of Fig. 16, but for C09io library of models.

UGC 08234 , that a contribution of 85 per cent of intermediate-age SSPs was required in the optical but only old SSPs were used in the NIR. When dealing with the reddening, these libraries also tend to overestimate the value of $A_{\mathrm{v}}$ by $\sim 1 \mathrm{mag}$.

The high-resolution models, on the other hand, perform considerably better, producing self-consistent fits from NIR and optical fits. The reddening found with MG15 models is almost the same for the two wavelength ranges for the whole sample, while MIUSCAT fits required an $A_{\mathrm{V}} 0.3$ mag larger for two objects. From the two last panels of Figs 22 and 23, it is possible to see that for both libraries, optical and NIR tend to find similar values of ${ }_{L}$ and MG15 tends to find lower values of $\langle Z\rangle_{L}$ in the NIR when compared to the optical.

Unfortunately, there are no libraries of high-resolution models that include SSPs younger than $1 \mathrm{Gyr}$, meaning that the full scenario cannot be tested. However, our results suggest that high-resolution models are essential to correctly disentangle the stellar population 

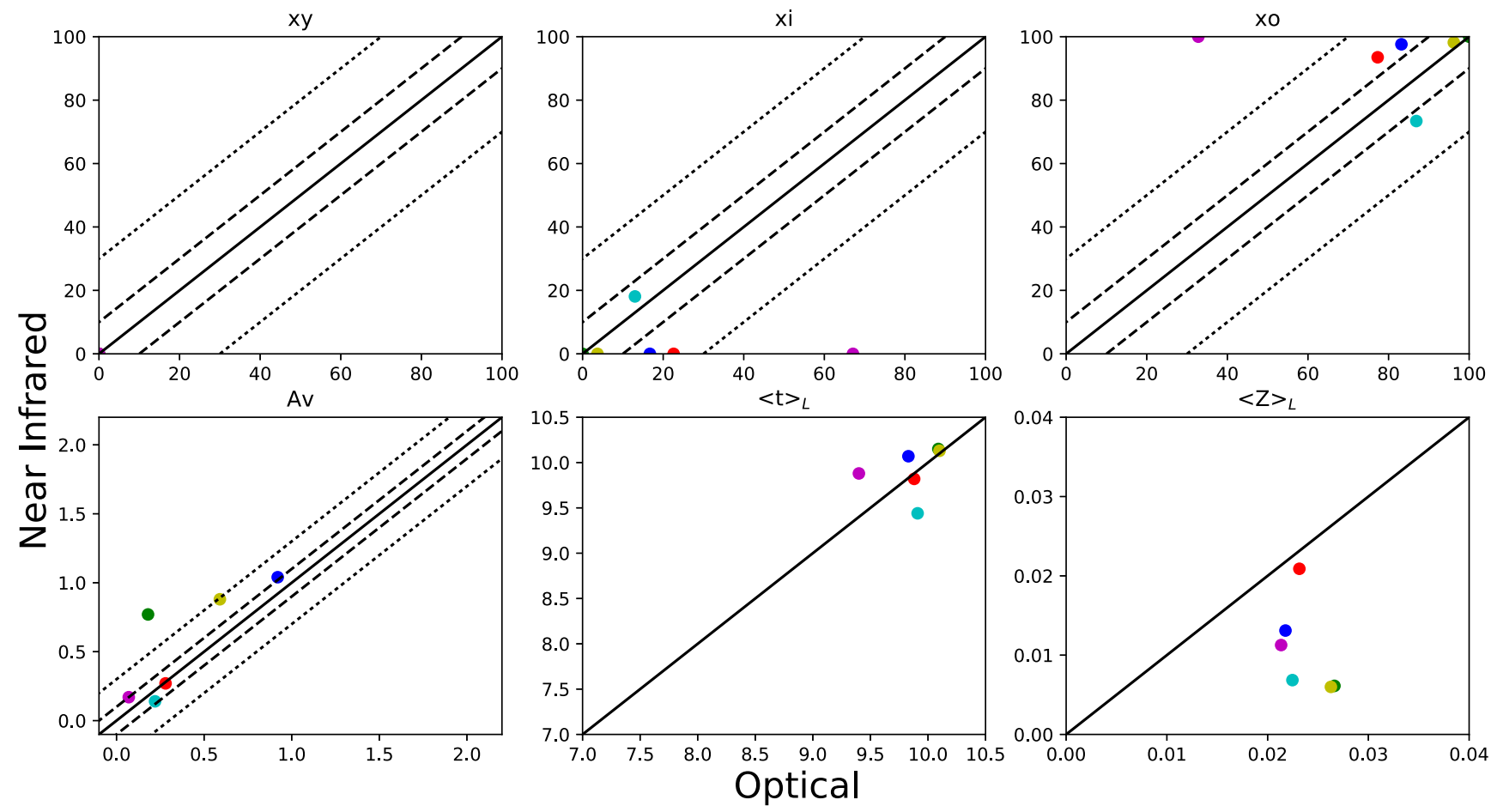

Figure 22. Same of Fig. 16, but for MG15 library of models.
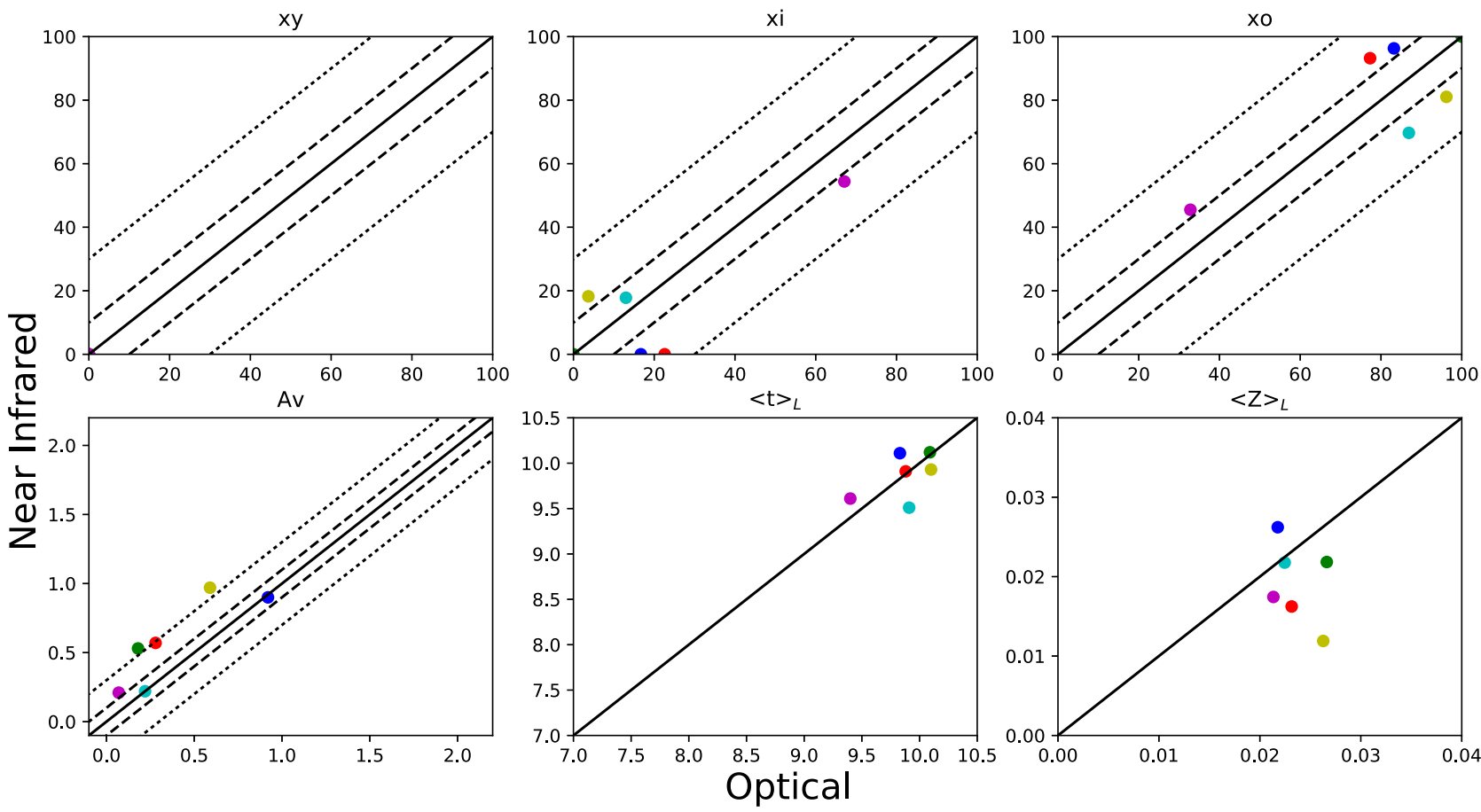

Figure 23. Same of Fig. 16, but for MIUSCAT library of models.

of a galaxy in the NIR, allowing in addition reliable reddening values. Otherwise, it is not possible to fit the absorptions and too much weight is given to the FC.

Baldwin et al. (2018) found that the impact of age variation in the NIR is largely dependent on the shape of the continuum. Our results show that the scenario is more complex, where the shape of the continuum is dependent on a combination of age and extinction, while the depth, shape, and width of the absorption features are crucially shaped by the age of the population. For low-resolution SSPs, these features will be diluted. As a result, STARLIGHT will have more difficulties to differentiate among the individual SSPs. Therefore, we reinforce the need of adequate NIR stellar libraries 
Table 5. Equivalent widths for the NIR absorption bands measured in $\AA$.

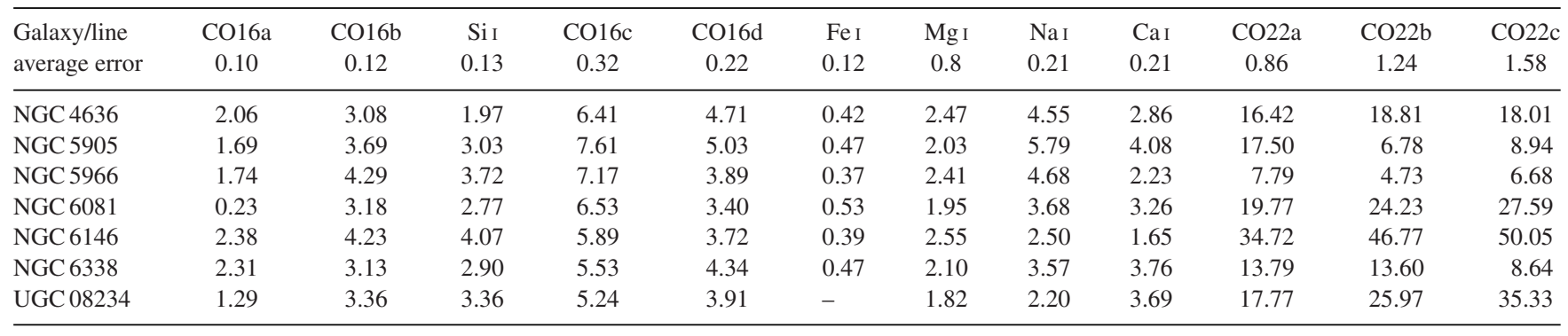

Table 6. Line limits and continuum bandpasses.

\begin{tabular}{lccc}
\hline $\begin{array}{l}\text { Centre } \\
(\AA)\end{array}$ & $\begin{array}{c}\text { Main } \\
\text { absorber }\end{array}$ & $\begin{array}{c}\text { Line limits } \\
(\AA)\end{array}$ & $\begin{array}{c}\text { Continuum bandpass } \\
(\AA)\end{array}$ \\
\hline 15587 & $\mathrm{CO}$ & $15555-15620$ & $15110-15170,15390-15410,16270-16310,16570-16580$ \\
15772 & $\mathrm{CO}$ & $15735-15810$ & $15110-15170,15390-15410,16270-16310,16570-16580$ \\
15890 & $\mathrm{Si}$ & $15850-15930$ & $15110-15170,15390-15410,16270-16310,16570-16580$ \\
16215 & $\mathrm{CO}$ & $16145-16285$ & $15110-15170,15390-15410,16270-16310,16570-16580$ \\
16385 & $\mathrm{CO}$ & $16340-16430$ & $15110-15170,15390-15410,16270-16310,16570-16580$ \\
17054 & $\mathrm{Fe}$ & $17025-17083$ & $16970-17083,17140-17200$ \\
17106 & $\mathrm{Mg}$ & $17083-17130$ & $21700-21930,22150-22200$ \\
22025 & $\mathrm{Na}$ & $21950-22100$ & $22450-22550,22680-22730$ \\
22620 & $\mathrm{Ca}$ & $22570-22670$ & $22690-22790,23655-23680,23890-23920$ \\
23015 & $\mathrm{CO}$ & $22870-23160$ & $22690-22790,23655-23680,23890-23920$ \\
23290 & $\mathrm{CO}$ & $23160-23420$ & $22690-22790,23655-23680,23890-23920$ \\
23535 & $\mathrm{CO}$ & $23420-23650$ &
\end{tabular}

Notes: The NIR indexes are based on Riffel et al. (2008, 2009, 2011, 2015).

for hotter stars, allowing thus the production of models with ages younger than 1 Gyr. This is specially important to properly fit the stellar content of active star-forming galaxies.

\subsection{Absorption band measurements}

For comparison with future NIR SP studies, we computed the equivalent widths $\left(W_{\lambda}\right)$ of the NIR absorption features measured with a PYTHON version of pacce code (Riffel \& Borges Vale 2011). The values of $\mathrm{W}_{\lambda}$ are presented on Table 5. They were calculated based on the line limits and continuum bandpasses of Table 6. Mild correlations were found between CO16a and $\mathrm{Mg}$ I, CO16b and S II, CO16c and $\mathrm{Na}$ I, CO16d, and $\mathrm{NaI}, \mathrm{Fe}$ I and $\mathrm{Ca}$, and the CO22 bands. Since the sample is small, these correlations should be seen with caution.

\section{FINAL REMARKS}

In this work, we compared the stellar population of a sample of six ETGs and one spiral galaxy, both in the optical and NIR spectral ranges. We chose for the NIR eight different bases of SSPs with different spectral resolutions and used different sets of isochrones to separately discuss their effects on the synthesis results. For four of the ETGs, we performed spectral synthesis in the optical using SDSS spectra and compared the results with those of the NIR. The approach followed here is based on the STARLIGHT code, which considers the whole observed spectrum, both the continuum and absorption features.

The main results can be summarized as follows: for spectral synthesis using bases with low spectral resolution, the results are more linked to the library of models used rather than to the object properties themselves. While $\mathrm{BC} 03$ models display a trend towards higher contributions of young populations, M05 usually finds more contributions from intermediate-age populations whereas C09 tend to find higher contributions from old stellar populations. Of all the cases, the values of Adev are compatible, meaning that none of these bases offer a more reliable result. When using bases with high spectral resolution, MG15 and MIUSCAT produced more consistent results if compared to low-spectral-resolution libraries. Out of the seven galaxies, the two libraries fitted consistent results for six of them.

The optical synthesis for the elliptical galaxies revealed a dominance of old stellar populations. The only exception was M05 library, which still found a high fraction of intermediate-age populations. This may indicate that the TP-AGB treatment plays an important role, even in the optical region.

When comparing optical and NIR results, we found that NIR fits using low spectral resolution libraries tend to overestimate the amount of young SSPs and the reddening. The only exception were the M05 models, which produced self-consistent fits, but predicted sizable amounts of intermediate-age stars for the galaxies. For libraries with high spectral resolution, since they do not include young SSPs, this scenario cannot be fully tested. However, the reddening found was compatible with literature. Also, high-spectral-resolution libraries produced results much more consistent if compared to models with low spectral resolution.

We tabulated the equivalent widths $\left(W_{\lambda}\right)$ of the NIR absorption features and the optical emission line fluxes to be used in future studies. Lastly, from the emission line ratios, we classified, for the first time in the literature, NGC 6081 and NGC 6338 as LINERs. 


\section{ACKNOWLEDGEMENTS}

LGDH thanks CAPES and CNPq. RR thanks CNPq and FAPERGS for partial funding of this project. ARA thanks CNPq for partial support to this work. LPM thanks CNPQ and FAPESP for partial funding of this project. CK acknowledges support through the research project AYA2017-79724-C4-4-P from the Spanish PNAYA. This research made use of the NASA/IPAC Extragalactic Database (NED), which is operated by the Jet Propulsion Laboratory, California Institute of Technology, under contract with the National Aeronautics and Space Administration. This study uses data provided by the Calar Alto Legacy Integral Field Area (CALIFA) survey (http://califa.caha.es/). Based on observations collected at the Centro Astronómico Hispano Alemán (CAHA) at Calar Alto, operated jointly by the Max-Planck-Institut für Astronomie and the Instituto de Astrofísica de Andalucía (CSIC). We thank the referee Reynier Peletier for carefully reading our paper and for giving such constructive comments which substantially helped improving the quality of the paper.

\section{REFERENCES}

Baldwin J. A., Phillips M. M., Terlevich R., 1981, PASP, 93, 5 (BPT)

Baldwin C. M., McDermid R. M., Kuntschner H., Maraston C., Conroy C., 2018, MNRAS, 473, 4698

Bamford S. P., Rojas A. L., Nichol R. C., Miller C. J., Wasserman L., Genovese C. R., Freeman P. E., 2008, MNRAS, 391, 607

Bruzual G., Charlot S., 2003, MNRAS, 344, 1000 (BC03)

Caccianiga A., Marchã M. J., Antón S., Mack K.-H., Neeser M. J., 2002, MNRAS, 329, 877

Capozzi D., Maraston C., Daddi E., Renzini A., Strazzullo V., Gobat R. 2016, MNRAS, 456, 790

Cardelli J. A., Clayton G. C., Mathis J. S., 1989, ApJ, 345, 245

Cassisi S., degl'Innocenti S., Salaris M., 1997a, MNRAS, 290, 515

Cassisi S., Castellani M., Castellani V., 1997b, A\&A, 317, 108

Chen X. Y., Liang Y. C., Hammer F., Prugniel P., Zhong G. H., Rodrigues M., Zhao Y. H., Flores H., 2010, A\&A, 515, A101

Cid Fernandes R., Gu Q., Melnick J., Terlevich E., Terlevich R., Kunth D., Rodrigues Lacerda R., Joguet B., 2004, MNRAS, 355, 273

Cid Fernandes R., Mateus A., Sodré L., Stasińska G., Gomes J. M., 2005 , MNRAS, 358, 363

Conroy C., 2013, ARA\&A, 51, 393

Conroy C., Gunn J. E., 2010, ApJ, 712, 833

Conroy C., Gunn J. E., White M., 2009, ApJ, 699, 486 (C09)

Cushing M. C., Vacca W. D., Rayner J. T., 2004, PASP, 116, 362

Cushing M. C., Rayner J. T., Vacca W. D., 2005, ApJ, 623, 1115

Dametto N. Z., Riffel R., Pastoriza M. G., Rodríguez-Ardila A., HernandezJimenez J. A., Carvalho E. A., 2014, MNRAS, 443, 1754

de Vaucouleurs G., de Vaucouleurs A., Corwin H. G., Jr., Buta R. J., Paturel G., Fouqué P., 1991, Third Reference Catalogue of Bright Galaxies. Volume I: Explanations and References. Volume II: Data for Galaxies between $0^{\mathrm{h}}$ and $12^{\mathrm{h}}$. Volume III: Data for Galaxies between $12^{\mathrm{h}}$ and $24^{\text {h }}$. Springer-Verlag, New York, NY

Dressel L. L., Condon J. J., 1978, ApJS, 36, 53

Forman W., Jones C., Tucker W., 1985, ApJ, 293, 102

Gomes J. M. et al., 2016, A\&A, 588, A68

Höfner S., Loidl R., Aringer B., Jørgensen U. G., Hron J., 2000, in Salama A., Kessler M. F., Leech K., Schulz B., eds, ESA SP-456, ISO Beyond the Peaks: The 2nd ISO Workshop on Analytical Spectroscopy, p. 299

Jones C., Forman W., Vikhlinin A., Markevitch M., David L., Warmflash A., Murray S., Nulsen P. E. J., 2002, ApJ, 567, L115

Kehrig C. et al., 2012, A\&A, 540, A11

Kriek M. et al., 2010, ApJ, 722, L64

Kroupa P., 2001, MNRAS, 322, 231

Lançon A., Mouhcine M., 2002, A\&A, 393, 167

Le Borgne J.-F. et al., 2003, A\&A, 402, 433

Lejeune T., Cuisinier F., Buser R., 1997, A\&AS, 125
Lejeune T., Cuisinier F., Buser R., 1998, A\&AS, 130, 65

Maraston C., 2005, MNRAS, 362, 799 (M05)

Maraston C., Strömbäck G., 2011, MNRAS, 418, 2785

Marigo P., Girardi L., Bressan A., Groenewegen M. A. T., Silva L., Granato G. L., 2008, A\&A, 482, 883

Martel A. R. et al., 2004, AJ, 128, 2758

Martins L. P., Lanfranchi G., Gonçalves D. R., Magrini L., Teodorescu A. M., Quireza C., 2012, MNRAS, 419, 3159

Martins L. P., Rodríguez-Ardila A., Diniz S., Gruenwald R., de Souza R., 2013, MNRAS, 431, 1823

Meneses-Goytia S., Peletier R. F., Trager S. C., Vazdekis A., 2015, A\&A, 582, A97 (MG15)

Nilson P., 1973, Uppsala General Catalogue of Galaxies. Astronomiska Observatorium, Uppsala

Noël N. E. D., Greggio L., Renzini A., Carollo C. M., Maraston C., 2013, ApJ, 772, 58

Padilla N. D., Strauss M. A., 2008, MNRAS, 388, 1321

Pandge M. B., Vagshette N. D., David L. P., Patil M. K., 2012, MNRAS, 421,808

Pietrinferni A., Cassisi S., Salaris M., Castelli F., 2004, ApJ, 612, 168

Raichur H., Das M., Herrero A. A., Shastri P., Kantharia N. G., 2015, Ap\&SS, 357, 32

Rayner J. T., Cushing M. C., Vacca W. D., 2009, ApJS, 185, 289

Rickes M. G., Pastoriza M. G., Bonatto C., 2009, A\&A, 505, 73

Riffel R., Borges Vale T., 2011, Ap\&SS, 334, 351

Riffel R., Pastoriza M. G., Rodríguez-Ardila A., Maraston C., 2007, ApJ, 659, L103

Riffel R., Pastoriza M. G., Rodríguez-Ardila A., Maraston C., 2008, MNRAS, 388, 803

Riffel R., Pastoriza M. G., Rodríguez-Ardila A., Bonatto C., 2009, MNRAS, 400, 273

Riffel R., Riffel R. A., Ferrari F., Storchi-Bergmann T., 2011, MNRAS, 416, 493

Riffel R. et al., 2015, MNRAS, 450, 3069

Röck B., Vazdekis A., Ricciardelli E., Peletier R. F., Knapen J. H., FalcónBarroso J., 2016, A\&A, 589, A73 (MIUSCAT)

Salpeter E. E., 1955, ApJ, 121, 161

Sánchez S. F. et al., 2012, A\&A, 538, A8

Sánchez S. F. et al., 2016, A\&A, 594, A36

Schaller G., Schaerer D., Meynet G., Maeder A., 1992, A\&AS, 96, 269

Schmidt A. A., Copetti M. V. F., Alloin D., Jablonka P., 1991, MNRAS, 249,766

Stanger V. J., Warwick R. S., 1986, MNRAS, 220, 363

Vacca W. D., Cushing M. C., Rayner J. T., 2004, PASP, 116, 352

Véron-Cetty M.-P., Véron P., 2006, A\&A, 455, 773

Walcher C. J. et al., 2014, A\&A, 569, A1

Westera P., Lejeune T., Buser R., Cuisinier F., Bruzual G., 2002, A\&A, 381, 524

Wilson J. C. et al., 2004, in Moorwood A. F. M., Iye M., eds, Proc. SPIE Conf. Ser. Vol. 5492, Ground-based Instrumentation for Astronomy. SPIE, Bellingham, p. 1295

Worthey G., 1994, ApJS, 95, 107

Wrobel J. M., 1984, ApJ, 284, 531

Zibetti S., Gallazzi A., Charlot S., Pierini D., Pasquali A., 2013, MNRAS, 428,1479

\section{SUPPORTING INFORMATION}

Supplementary data are available at MNRAS online.

Please note: Oxford University Press is not responsible for the content or functionality of any supporting materials supplied by the authors. Any queries (other than missing material) should be directed to the corresponding author for the article. 


\section{APPENDIX A: INDIVIDUAL DESCRIPTION OF THE SAMPLE}

\section{A1 NGC 4636}

NGC 4636 is a giant elliptical galaxy with LINER emission. It is one of the nearest and more X-ray luminous ellipticals, with $L_{X}=2 \times 10^{41} \mathrm{ergs} \cdot \mathrm{s}^{-1}$ (Jones et al. 2002). It is surrounded by an extended corona of hot gas (Forman, Jones \& Tucker 1985), and has an asymmetric gas distribution, probably the result of irregular flows (Stanger \& Warwick 1986).

\section{A2 NGC 5905}

This galaxy is one of a few that did not have a previous evidence of an AGN but that had X-ray eruptions observed, what happened in 1991 and 1992 with Chandra telescope. Later, X-ray flux started to decrease in a rate consistent with the expected rate for a tidal disruption event (TDE). Recent observations showed that the infrared flux is dominated by star formation, which suggests that the radio emission is caused by circumnuclear star formation. Besides, no radio emission consistent with the TDE event was detected (Raichur et al. 2015). Among the galaxies of our sample, it is the only galaxy that has clearly visible NIR emission lines and the only non-ETG.

\section{A3 NGC 5966}

NGC 5966 is an elliptical galaxy with a faint bar-like feature centred at the nucleus. Stars and gas are decoupled, with the gas showing an elongated emission feature, in such a way that an ionization cone or a decoupled rotational disc are two possible interpretations (Kehrig et al. 2012). According to these authors, the diagnostic diagrams indicate the presence of a LINER nucleus and a LINERlike gas emission extending $\sim 5 \mathrm{kpc}$ outward from the nucleus, also LINER-like. The presence of a nuclear ionizing source seems to be required to shape the elongated gas emission feature in the 'ionization cone' scenario, although ionization by pAGB stars cannot be ruled out. On the other hand, Gomes et al. (2016) classified this object as type i ETG, also reporting that the absence of ongoing star formation throughout the galaxy lends support to the idea that its gas outflow is powered by an AGN hosted in its LINER nucleus. According to Gomes et al. (2016), a type i ETG is a system with a nearly constant $\mathrm{EW}(\mathrm{H} \alpha)$ in their extranuclear component, compatible with the hypothesis of photoionization by pAGB as the main driver of extended warm interstellar medium (WIM) emission.

\section{A4 NGC 6081}

It is a galaxy with radio emission (Dressel \& Condon 1978). Also, Gomes et al. (2016) classified it as a type i ETG, reporting that sources other than pAGB stars dominate in less than 7 percent of the area of the galaxy. Its SDSS spectra is dominated by stellar population, with strong absorption bands and [O III], [N $\mathrm{I}], \mathrm{H} \alpha$, and $\left[\mathrm{S}_{\mathrm{II}}\right.$ ] typical of LINERs. Its APO spectrum shows a stellar continuum dominated source with strong $\mathrm{CO}$ bands and absence of emission lines.

\section{A5 NGC 6146}

NGC 6146 is an elliptical galaxy with a radio jet (Wrobel 1984). Caccianiga et al. (2002) reported the detection of $\mathrm{Ca}$ II $\mathrm{H}$ and $\mathrm{K}$, $\mathrm{G}, \mathrm{Mg} \mathrm{Ib}$, and $\mathrm{Na}$ Id absorption bands and a weak $\mathrm{H} \alpha$ emission line. Gomes et al. (2016) classified this galaxy as type i ETG, also reporting that the absence of star formation and the suggestion of an outflow are compatible with the idea of a low luminosity AGN powering the nucleus. Its APO spectra shows a stellar continuum dominated source with strong $\mathrm{CO}$ bands and absence of emission lines.

\section{A6 NGC 6338}

This galaxy is the brightest on Draco constellation. It possesses diffused and ionized gas and dust filaments on kiloparsec scales (Martel et al. 2004). X-ray observations indicate two or possibly three emission cavities of ellipsoidal shape with lower X-ray surface flux. They also found cold filamentary structures matching the $\mathrm{H} \alpha$ emission and high extinction regions seen on the optical extinction maps. This indicates a cooling mechanism generated by dust (Pandge et al. 2012). On the same work, they reported that a harder ionizing source is required to maintain such a high degree of ionization and that most of the jet power is mechanical. Gomes et al. (2016), using CALIFA data cubes, classified this object as type ii ETG.

\section{A7 UGC 08234}

This galaxy is part of a galaxy group and possesses a spiral companion, UGC 08237 (Nilson 1973). Gomes et al. (2016) classified this object as type ii ETG. There are no X-ray or radio properties reported in the literature. Its APO spectrum is dominated by stellar population, characterized by intense absorption bands and absence of emission lines.

\section{APPENDIX B: EMISSION LINE FLUXES}

In the optical region, according to Baldwin, Phillips \& Terlevich (1981, hereafter BPT), the line ratios [O III] $\lambda 5007 \AA / \mathrm{H} \beta$ and $[\mathrm{N}$ II $] \lambda 6583 \AA / \mathrm{H} \alpha$ can be used to classify the excitation mechanism that powers the optical lines based on the position of the points in the diagram. After running the stellar population synthesis for the SDSS spectra, we subtracted the stellar component and measured the emission line fluxes by fitting Gaussians to the emission lines. Although there are still controversies on individual objects (e.g. Martins et al. 2012, who found a galaxy with no signs of activity but whose line ratios put it as a Seyfert galaxy), these diagrams can be used with great confidence to study the nature of the central source that powers the observed emission lines.

The emission line fluxes measured in the galaxy sample are listed in Table B1 for the NIR and Table B2 for the optical. Also, the $\left[\mathrm{O}{ }_{\mathrm{III}}\right] / \mathrm{H} \beta$ and $\left[\mathrm{N}_{\mathrm{II}}\right] / \mathrm{H} \alpha$ ratios are plotted in Fig. B1. From the locus of points occupied by our targets, we confirm the LINER nature of the galaxies. Only NGC 4636 and NGC 5966 had already been previously classified as LINER (Véron-Cetty \& Véron 2006; Kehrig et al. 2012). The results obtained allow us to state that our sample is composed of five ETGs with LINER emission, one spiral galaxy, and one ETG with no data about its emission lines. 
Table B1. NIR emission line fluxes measured for NGC 5905.

\begin{tabular}{lcc}
\hline Line & $\begin{array}{c}\text { Flux } \\
\left(\times 10^{-15} \mathrm{ergs} \mathrm{s}^{-1} \mathrm{~cm}^{-2}\right)\end{array}$ & $\begin{array}{c}\text { FWHM } \\
\left(\mathrm{km} \mathrm{s}^{-1}\right)\end{array}$ \\
\hline$[\mathrm{Fe}$ II $] \lambda 12570 \AA$ & 9.33 & 806 \\
$\mathrm{~Pa} \beta$ & 6.72 & 315 \\
$\mathrm{H}_{2} \lambda 21218 \AA$ & 2.68 & 385 \\
$\mathrm{Br} \gamma$ & 3.5 & 462 \\
\hline
\end{tabular}

Table B2. Optical emission line fluxes in units of $10^{-15} \mathrm{ergs} \mathrm{s}^{-1} \mathrm{~cm}^{-2} \AA^{-1}$.

\begin{tabular}{llllllll}
\hline Object & $\mathrm{H} \beta$ & {$[\mathrm{O} \mathrm{III}]$} & {$[\mathrm{N} \mathrm{II}]$} & $\mathrm{H} \alpha$ & {$[\mathrm{N} \mathrm{II}]$} & {$\left[\mathrm{S}_{\mathrm{II}}\right]$} & {$\left[\mathrm{S}_{\mathrm{II}}\right]$}
\end{tabular}
$4861 \AA 5007 \AA 6549 \AA 6563 \AA 6585 \AA 6718 \AA 6732 \AA$

\begin{tabular}{lllllrll}
\hline NGC 4636 & 1.9 & 2.7 & 3.43 & 6.84 & 8.77 & 3.48 & 3.10 \\
NGC 5966 & 0.87 & 2.26 & 1.75 & 2.69 & 3.92 & 2.79 & 2.00 \\
NGC 6081 & 1.05 & 3.7 & 7.26 & 6.73 & 13.5 & 5.61 & 3.71 \\
NGC 6338 & 1.82 & 2.38 & 4.37 & 5.43 & 11.5 & 3.99 & 2.87 \\
\hline
\end{tabular}

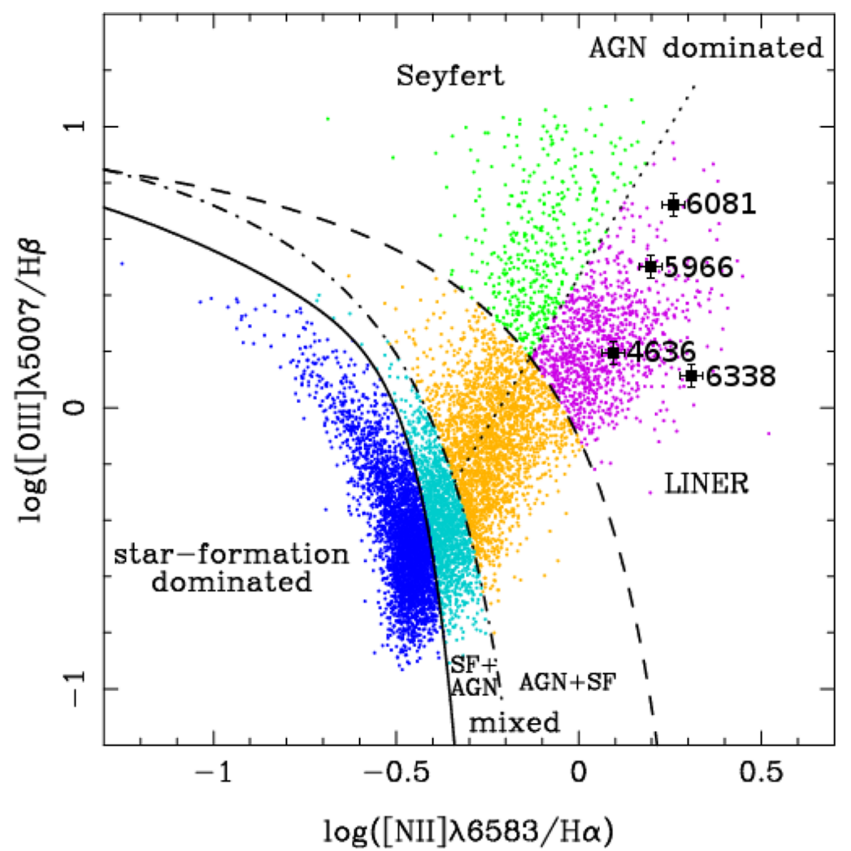

Figure B1. BPT diagram for the galaxies with optical spectra. The number of the NGC catalogue is shown on the right-hand side of each point. The points were plotted over the diagram from Bamford et al. (2008)

This paper has been typeset from a $\mathrm{T}_{\mathrm{E}} \mathrm{X} / \mathrm{LT}_{\mathrm{E}} \mathrm{X}$ file prepared by the author. 